\title{
Semiempirical formula for electroweak response functions in the two-nucleon emission channel in neutrino-nucleus scattering
}

\author{
V. L. Martinez-Consentino $\oplus^{*}$ J. E. Amaro,$^{\dagger}$ and I. Ruiz Simo $\circledast^{\ddagger}$ \\ Departamento de Física Atómica, Molecular y Nuclear and Instituto Carlos I de Física \\ Teórica y Computacional Universidad de Granada, E-18071 Granada, Spain
}

(Received 3 September 2021; accepted 2 December 2021; published 27 December 2021)

\begin{abstract}
A semiempirical formula for the inclusive electroweak response functions in the two-nucleon emission channel is proposed. The method consists in expanding each one of the vector-vector, axial-axial, and vector-axial responses as sums of six subresponses. These correspond to separating the meson-exchange currents as the sum of three currents of similar structure and expanding the hadronic tensor as the sum of the separate contributions from each current plus the interferences between them. For each subresponse, we factorize the coupling constants, the electroweak form factors, the phase space, and the delta propagator, for the delta-forward current. The remaining spin-isospin contributions are encoded in coefficients for each value of the momentum transfer, $q$. The coefficients are fitted to the exact results in the relativistic mean field model of nuclear matter, for each value of $q$. The dependence on the energy transfer $\omega$ is well described by the semiempirical formula. The $q$-dependency of the coefficients of the subresponses can be parametrized or can be interpolated from the provided tables. The description of the five theoretical responses is quite good. The parameters of the formula, the Fermi momentum, number of particles, relativistic effective mass, vector energy, the electroweak form factors, and the coupling constants, can be modified easily. This semiempirical formula can be applied to the cross section of neutrinos, antineutrinos, and electrons.
\end{abstract}

DOI: 10.1103/PhysRevD.104.113006

\section{INTRODUCTION}

In recent years, the study of quasielastic neutrino scattering by nuclei has received increasing interest from the theoretical and experimental point of view. This is due to recent neutrino accelerator experiments aimed at measuring neutrino-nucleus cross sections. The ultimate goal is to measure the neutrino oscillation parameters accurately, something that requires knowing with precision the cross sections of neutrino scattering with the nuclei of the detectors. Extensive reviews describing the state of the art of the subject, namely, recent advances and open challenges in the field of neutrino-nucleus scattering, can be found in Refs. [1-9].

Experimental measurements of the inclusive chargechanging (CC) quasielastic cross section $\left(\nu_{\mu}, \mu^{-}\right)$and $\left(\bar{\nu}_{\mu}, \mu^{+}\right)$, have been carried out in several laboratories, each of which is characterized by a neutrino flux or distribution in a range of energies with different widths around $1 \mathrm{GeV}[10$ 16]. Comparison of these data with the different nuclear models of the reaction revealed the discrepancies between the different approaches, as well as discrepancies with the

\footnotetext{
victormc@ugr.es

amaro@ugr.es

ruizsig@ugr.es
}

experimental data [17-25]. In particular, the importance of including the contributions of multinucleon emission was highlighted. The calculation of two-particles two-holes $(2 \mathrm{p} 2 \mathrm{~h})$ contribution varies in different models, but all agree on a contribution that can be around $20 \%$ of the cross section with one-nucleon emission only.

Neutrino CC cross sections are averages for different incident energies. Therefore, the differences between models are also differences between averages-although theoretically, the double differential cross section is calculated for fixed neutrino energies. To compare with data for fixed energy, the only possibility is to use the electron $\left(e, e^{\prime}\right)$ experiments. This allows the neutrino models to be calibrated, taking into account that the electromagnetic current is related to the vector part of the weak current. It is only the axial current that is not fixed by the electrons. Various approaches to the study of quasielastic lepton scattering have been applied. For finite nuclei quantum Monte Carlo methods [26], continuum shell model with random phase approximation (RPA) correlations [27], or spectral function methods $[28,29]$ can be mentioned. For high or intermediate energies, relativistic corrections are important, yet corrections to kinetic energy can be partially included. But the nuclear interaction at the relativistic level requires a special care $[30,31]$. The relativistic mean field (RMF) is the simplest model available that includes the 
interaction in a fully relativistic way in the form of scalar and vector mean potentials [32,33]. Other models use fits to generate, e.g., parametrizations of the transverse enhancement of the transverse response [19,34]. Most of the approaches require approximations that sometimes violate fundamental current conservation requirements. The influence of other factors such as short-range correlations, finalstate interaction, exchange currents, pion emission, and resonance excitation contribute to the differences between the various models and to the difficulty of accurately reproducing electron data in the full kinematical range [35].

In this paper, we focus on the inclusive two-nucleon emission $(2 \mathrm{p} 2 \mathrm{~h})$ channel for neutrino and electron scattering. Because of the nuclear force between correlated nucleons, a high-energy neutrino can induce emission of a pair of nucleons. In the case of $\left(\nu_{\mu}, \mu^{-}\right)$reaction, protonproton (PP) and proton-neutron (PN) pairs can be ejected, while neutron-neutron (NN) and $\mathrm{PN}$ pairs can be ejected in the $\left(\bar{\nu}_{\mu}, \mu^{+}\right)$reaction. A large part of the $2 \mathrm{p} 2 \mathrm{~h}$ cross section is produced by interaction with nuclear meson-exchange currents (MECs), where the exchanged bosons $W^{ \pm}$are absorbed by mesons exchanged between two nucleons. The most important part of the interaction occurs when the boson absorption by the nucleon produces a virtual $\Delta$ that decays and communicates its energy to a pair of nucleons.

Several phenomenological models have been proposed to calculate the $2 \mathrm{p} 2 \mathrm{~h}$ channel in neutrino interactions. Each of these models, based on the Fermi gas, takes its own approximations, and in general they are numerically expensive. A first calculation revealed the importance of $2 \mathrm{p} 2 \mathrm{~h}$ to reproduce the data of neutrino scattering $[17,36]$, and similar effects were found in a relativistic calculation $[37,38]$ and also in a local Fermi gas with RPA and other many-body corrections $[18,39,40]$. These models require integration in many dimensions and intensive numerical computation of traces of two-body operators. Although several approaches have been proposed $[41,42]$ that reduce the calculation, in the absence of analytical formulas for the $2 \mathrm{p} 2 \mathrm{~h}$ responses, it is necessary to elaborate numerical tables or parametrizations of theoretical calculations for their implementation in Monte Carlo simulators $[43,44]$.

In Ref. [45], the impact of $2 \mathrm{p} 2 \mathrm{~h}$ was investigated in a simple model using an ansatz physically motivated by the phase-space function of two particles in a Fermi gas. The matrix elements of the MECs averaged over the neutrino flux were approximated by a constant that was fitted to the neutrino data. In this work, we explore extensions of this simple model by adding other physical dependencies, motivated by the theoretical structure of the MEC operators, which contain form factors, coupling constants, and propagators of the $\Delta$, as physical dependencies that must be present in the MEC responses. The rest of the more complex dependencies associated with elements of the spin-isospin matrix of Dirac operators are synthesized in constants for each value of the transferred momentum.
These constants are obtained by a fit of a theoretical model but could also be fitted to data if there were a sufficient number of them. The resulting semiempirical (SE) formula for the MEC responses allows us to compute the inclusive $2 \mathrm{p} 2 \mathrm{~h}$ responses analytically for fixed $q$, having the parameters tabulated as a function of $q$, and to interpolate for intermediate values.

The need of an analytical formula for the inclusive $2 \mathrm{p} 2 \mathrm{~h}$ responses is based on the difficulty of calculating the integral in seven dimensions for the $2 \mathrm{p} 2 \mathrm{~h}$ responses, which then has to be integrated with the neutrino flux. With a suitable parametrization, the calculation time is reduced. An alternative parametrization was carried out in the relativistic Fermi gas (RFG) model of Ref. [46] for the vector responses, containing Gaussians and polynomials. A similar parametrization was made in Ref. [20] for the axial contributions. In this paper, we follow a different approach, using the RMF model of nuclear matter and a semiempirical parametrization of the $2 \mathrm{p} 2 \mathrm{~h}$ responses that has the advantage that the dependence on the momentum, $q$, and energy transfer, $\omega$, has a theoretical basis. This is analogous to the Bethe-Weizsacker semiempirical mass formula of nuclear binding energy [47], where the dependence on $\mathrm{A}$ and $\mathrm{Z}$ also has a theoretical basis. In particular, we extract the dependence of the responses on the number of particles, the Fermi momentum, and the relativistic effective mass. Furthermore, the dependence is exact on the form factors and the electroweak coupling constants. The formula will contain constants that we will adjust to the exact results. The frozen and modified convolution approximations have been proposed $[41,42]$ that reduce the integrals to one dimension and five dimensions, respectively, to which an integral over the neutrino flux would have to be added. However, the semiempirical formula has the advantage that it is analytical and gives better results than the frozen one, since the parameters have been adjusted to reproduce the exact responses.

Note that the developments in this paper are applicable to the calculation of inclusive cross sections only. The MEC model that we use to fit the SE-MEC responses is based on Feynman diagrams for pion production on the nucleon. In our case, these diagrams will be considered in a RMF model of nuclear matter. In this model, the nucleons are relativistic and interact with vector and scalar potentials, the effect of which is to give them an effective mass and a repulsive vector energy. The idea is to combine this SEMEC formula with the superscaling approach with relativistic effective mass $M^{*}\left(\mathrm{SuSAM}^{*}\right)$ in which a new scaling function $f^{*}\left(\psi^{*}\right)$ including dynamical relativistic effects has been proposed [48-50] through the introduction of an effective mass for the nucleon. The SuSAM* model describes a large amount of the electron data within a phenomenological quasielastic band, and it was extended to the neutrino and antineutrino sector [51], SuSAM* was first developed from the set of ${ }^{12} \mathrm{C}$ data $[48,49]$ and later 
applied to other nuclei in Refs. [50,52]. Recently, the superscaling function has been refitted by subtraction of the MEC $2 \mathrm{p} 2 \mathrm{~h}$ cross section before performing the scaling analysis, in order to avoid double-counting when adding the MEC responses [53].

The scheme of the paper is as follows. In Sec. II, we present the formalism for neutrino scattering, the inclusive $2 \mathrm{p} 2 \mathrm{~h}$ hadronic tensor, the model of MEC, the analytical approximation of the phase-space integral in frozen nucleon approximation, and the averaged $\Delta$ propagator. In Sec. III, we write the semiempirical formula for the MEC responses. In Sec. IV, we present the results of the fit and compare with the exact results. In Sec. V, we draw our conclusions.

\section{FORMALISM OF CC NEUTRINO SCATTERING}

\section{A. Neutrino cross section}

In this section, we summarize the formalism for chargechanging neutrino scattering. The case of electron scattering can be easily inferred from this by considering only the longitudinal and transverse response functions. Thus, we consider charged-current inclusive quasielastic reactions in nuclei induced by neutrinos and antineutrinos, focusing on the $\left(\nu_{\mu}, \mu^{-}\right)$and $\left(\bar{\nu}_{\mu}, \mu^{+}\right)$cross sections. The relativistic energies of the incident (anti)neutrino and detected muon are $\epsilon=E_{\nu}$, and $\epsilon^{\prime}=m_{\mu}+T_{\mu}$, respectively. Their momenta are $\mathbf{k}$ and $\mathbf{k}^{\prime}$. The 4-momentum transfer is $k^{\mu}-k^{\prime \mu}=$ $Q^{\mu}=(\omega, \mathbf{q})$, with $Q^{2}=\omega^{2}-q^{2}<0$. The lepton scattering angle, $\theta$, is the angle between $\mathbf{k}$ and $\mathbf{k}^{\prime}$. The doubledifferential cross section can be written as

$$
\begin{aligned}
\frac{d^{2} \sigma}{d T_{\mu} d \cos \theta}= & \frac{G^{2} \cos ^{2} \theta_{c}}{4 \pi} \frac{k^{\prime}}{\epsilon} v_{0}\left[V_{C C} R_{C C}+2 V_{C L} R_{C L}\right. \\
& \left.+V_{L L} R_{L L}+V_{T} R_{T} \pm 2 V_{T^{\prime}} R_{T^{\prime}}\right] .
\end{aligned}
$$

Inside the brackets in Eq. (1), there is a linear combination of the five nuclear response functions, where $(+)$ is for neutrinos and ( - ) is for antineutrinos. Here, $G=1.166 \times$ $10^{-11} \mathrm{MeV}^{-2}$ is the Fermi constant, $\theta_{c}$ is the Cabibbo angle, $\cos \theta_{c}=0.975$, and the kinematical factor $v_{0}=\left(\epsilon+\epsilon^{\prime}\right)^{2}-q^{2}$. The $V_{K}$ coefficients depend only on the lepton kinematics and do not depend on the details of the nuclear target,

$$
\begin{gathered}
V_{C C}=1+\delta^{2} \frac{Q^{2}}{v_{0}} \\
V_{C L}=\frac{\omega}{q}-\frac{\delta^{2}}{\rho^{\prime}} \frac{Q^{2}}{v_{0}} \\
V_{L L}=\frac{\omega^{2}}{q^{2}}-\left(1+\frac{2 \omega}{q \rho^{\prime}}+\rho \delta^{2}\right) \delta^{2} \frac{Q^{2}}{v_{0}}
\end{gathered}
$$

$$
\begin{gathered}
V_{T}=-\frac{Q^{2}}{v_{0}}+\frac{\rho}{2}+\frac{\delta^{2}}{\rho^{\prime}}\left(\frac{\omega}{q}+\frac{1}{2} \rho \rho^{\prime} \delta^{2}\right) \frac{Q^{2}}{v_{0}} \\
V_{T^{\prime}}=-\frac{1}{\rho^{\prime}}\left(1-\frac{\omega \rho^{\prime}}{q} \delta^{2}\right) \frac{Q^{2}}{v_{0}},
\end{gathered}
$$

where we have defined the dimensionless factors $\delta=m_{\mu} / \sqrt{\left|Q^{2}\right|}$, proportional to the muon mass $m_{\mu}$, $\rho=\left|Q^{2}\right| / q^{2}$, and $\rho^{\prime}=q /\left(\epsilon+\epsilon^{\prime}\right)$.

The response functions, $R^{K}(q, \omega)$, are defined as suitable combinations of the hadronic tensor, $W^{\mu \nu}$, in a reference frame where the $z$ axis $(\mu=3)$ points along the momentum transfer $\mathbf{q}$ and the $x$ axis $(\mu=1)$ is defined as the transverse (to q) component of the (anti)neutrino momentum $\mathbf{k}$ lying in the lepton scattering plane; the $y$ axis $(\mu=2)$ is then normal to the lepton scattering plane. The usual components are then

$$
\begin{gathered}
R^{C C}=W^{00} \\
R^{C L}=-\frac{1}{2}\left(W^{03}+W^{30}\right) \\
R^{L L}=W^{33} \\
R^{T}=W^{11}+W^{22} \\
R^{T^{\prime}}=-\frac{i}{2}\left(W^{12}-W^{21}\right) .
\end{gathered}
$$

The inclusive hadronic tensor is constructed from the matrix elements of the current operator $J^{\mu}(Q)$ between the initial and final hadronic states, summing over all the possible final nuclear states with excitation energy $\omega=$ $E_{f}-E_{i}$ and averaging over the initial spin components,

$W^{\mu \nu}=\sum_{f} \overline{\sum_{i}}\left\langle f\left|J^{\mu}(Q)\right| i\right\rangle^{*}\left\langle f\left|J^{\nu}(Q)\right| i\right\rangle \delta\left(E_{i}+\omega-E_{f}\right)$.

In the case of electron scattering, the cross section is

$$
\frac{d \sigma}{d \Omega d \epsilon^{\prime}}=\sigma_{\mathrm{Mott}}\left(v_{L} R_{e m}^{L}+v_{T} R_{e m}^{T}\right) .
$$

where $\sigma_{\text {Mott }}$ is the Mott cross section, $v_{L}$ and $v_{T}$ are kinematic factors

$$
\begin{gathered}
v_{L}=\frac{Q^{4}}{q^{4}} \\
v_{T}=\tan ^{2} \frac{\theta}{2}-\frac{Q^{2}}{2 q^{2}} .
\end{gathered}
$$


The electromagnetic longitudinal and transverse response functions, $R_{e m}^{L}(q, \omega)$ and $R_{e m}^{T}(q, \omega)$, are

$$
\begin{gathered}
R_{e m}^{L}=W_{e m}^{00} \\
R_{e m}^{T}=W_{e m}^{11}+W_{e m}^{22} .
\end{gathered}
$$

Attending to the kind of final states, the hadronic tensors can be written as sum of one-particle (1p1h) plus twoparticles $(2 \mathrm{p} 2 \mathrm{~h}), \ldots$, emission channels

$$
W^{\mu \nu}=W_{1 \mathrm{p} 1 \mathrm{~h}}^{\mu \nu}+W_{2 \mathrm{p} 2 \mathrm{~h}}^{\mu \nu}+\cdots .
$$

In this work, we are interested in the $2 \mathrm{p} 2 \mathrm{~h}$ channel only. We studied the $1 \mathrm{p} 1 \mathrm{~h}$ responses and cross section for CC neutrino and antineutrino scattering in Ref. [51] and for electron scattering in Refs. [50,52,53]. Here, we extend these works to deepen the study of the interaction of neutrinos with nuclei at intermediate energies and the role of the $2 \mathrm{p} 2 \mathrm{~h}$ electroweak response functions, as described in the next subsection.

\section{B. 2p2h hadronic tensor in the RMF}

In this paper, we consider the $2 \mathrm{p} 2 \mathrm{~h}$ responses within the RMF model of nuclear matter [54-57]. In this model, the nucleons are interacting with a relativistic field containing scalar and vector potentials. The single-particle wave functions are plane waves with momentum $\mathbf{h}$ and with on-shell energy

$$
E=\sqrt{\left(m_{N}^{*}\right)^{2}+h^{2}}
$$

where $m_{N}^{*}$ is the relativistic effective mass of the nucleon defined by

$$
m_{N}^{*}=m_{N}-g_{s} \phi_{0}=M^{*} m_{N},
$$

where $m_{N}$ is the bare nucleon mass, $g_{s} \phi_{0}$ is the scalar potential energy of the RMF [55], and $M^{*}=0.8$ for ${ }^{12} \mathrm{C}$, the nucleus considered in our results. Additionally, the nucleon acquires a positive energy due to the repulsion by the relativistic vector potential, $E_{v}=g_{v} V_{0}$. Thus, the total nucleon energy is

$$
E_{\mathrm{RMF}}=E+E_{v} .
$$

In this work, we use values of $k_{F}, M^{*}$ and $E_{v}$ that are obtained phenomenologically from the data of $\left(e, e^{\prime}\right)$ [53]. In particular, we use the value $E_{v}=141 \mathrm{MeV}$ for ${ }^{12} \mathrm{C}$. In the semiempirical formulas in the next section, these values could easily be modified if desired. For many observables, that only depend on the energy differences between a final particle and an initial one, the vector energy cancels out and does not affect the results.
The nuclear states in the RMF are Slater determinants constructed with plane waves obtained by solving the free Dirac equation with effective mass $m_{N}^{*}$. Note that we use the same effective mass for particles and holes. In this approximation, the model is the simplest possible that implements the mean field, so the results for very large momenta should be taken with caution.

All states with momentum $h<k_{F}$ are occupied in the ground state, with $k_{F}$ the Fermi momentum. The $2 \mathrm{p} 2 \mathrm{~h}$ excitations are obtained by raising two particles above the Fermi level, with momenta $p_{1}^{\prime}$ and $p_{2}^{\prime}>k_{F}$, leaving two holes with momenta $h_{1}$ and $h_{2}<k_{F}$. In this work, we focus on the $2 \mathrm{p} 2 \mathrm{~h}$ part of the hadronic tensor. It is generated by the interaction with a two-body current operator, whose matrix elements are given by

$$
\begin{aligned}
\left\langle f\left|J^{\mu}(Q)\right| i\right\rangle= & \frac{(2 \pi)^{3}}{V^{2}} \delta\left(\mathbf{p}_{1}^{\prime}+\mathbf{p}_{2}^{\prime}-\mathbf{q}-\mathbf{h}_{1}-\mathbf{h}_{2}\right) \\
& \times \frac{\left(m_{N}^{*}\right)^{2}}{\sqrt{E_{1}^{\prime} E_{2}^{\prime} E_{1} E_{2}}} j^{\mu}\left(\mathbf{p}_{1}^{\prime}, \mathbf{p}_{2}^{\prime}, \mathbf{h}_{1}, \mathbf{h}_{2}\right),
\end{aligned}
$$

where $V$ is the volume of the system and the on-shell energies and momenta of the particles and holes are $\left(E_{i}^{\prime}, \mathbf{p}_{i}^{\prime}\right)$, and $\left(E_{i}, \mathbf{h}_{i}\right)$, for $i=1,2$. Note that 3 -momentum is conserved and that the relativistic factors $\left(m_{N}^{*} / E\right)^{1 / 2}$ contain the effective mass and the on-shell energies. The spin-isospin two-body current matrix elements $j^{\mu}\left(\mathbf{p}_{1}^{\prime}, \mathbf{p}_{2}^{\prime}, \mathbf{h}_{1}, \mathbf{h}_{2}\right)$ are defined in the next subsection.

Inserting this expression into the hadronic tensor, Eq. (12), and taking the limit $V \rightarrow \infty$ (infinite nuclear matter), we transform the sums into integrals, to obtain the $2 \mathrm{p} 2 \mathrm{~h}$ hadronic tensor for the RMF theory of nuclear matter

$$
\begin{aligned}
W_{2 \mathrm{p} 2 \mathrm{~h}}^{\mu \nu}= & \frac{V}{(2 \pi)^{9}} \int d^{3} p_{1}^{\prime} d^{3} p_{2}^{\prime} d^{3} h_{1} d^{3} h_{2} \\
& \times \frac{\left(m_{N}^{*}\right)^{4}}{E_{1} E_{2} E_{1}^{\prime} E_{2}^{\prime}} w^{\mu \nu}\left(\mathbf{p}_{1}^{\prime}, \mathbf{p}_{2}^{\prime}, \mathbf{h}_{1}, \mathbf{h}_{2}\right) \\
& \times \Theta\left(p_{1}^{\prime}, h_{1}\right) \Theta\left(p_{2}^{\prime}, h_{2}\right) \delta\left(E_{1}^{\prime}+E_{2}^{\prime}-E_{1}-E_{2}-\omega\right) \\
& \times \delta\left(\mathbf{p}_{1}^{\prime}+\mathbf{p}_{2}^{\prime}-\mathbf{q}-\mathbf{h}_{1}-\mathbf{h}_{2}\right),
\end{aligned}
$$

where $V /(2 \pi)^{3}=Z /\left(\frac{8}{3} \pi k_{F}^{3}\right)$ for symmetric nuclear matter. The Pauli blocking function $\Theta$ is defined as the product of step functions for the initial and final momentum

$$
\Theta\left(p_{i}^{\prime}, h_{i}\right) \equiv \theta\left(p_{i}^{\prime}-k_{F}\right) \theta\left(k_{F}-h_{i}\right) .
$$

Finally, the function $w^{\mu \nu}\left(\mathbf{p}_{1}^{\prime}, \mathbf{p}_{2}^{\prime}, \mathbf{h}_{1}, \mathbf{h}_{2}\right)$ represents the hadron tensor for a single $2 \mathrm{p} 2 \mathrm{~h}$ transition, summed up over spin and isospin, 


$$
\begin{aligned}
w^{\mu \nu} & \left(\mathbf{p}_{1}^{\prime}, \mathbf{p}_{2}^{\prime}, \mathbf{h}_{1}, \mathbf{h}_{2}\right) \\
\quad= & \frac{1}{4} \sum_{s_{1} s_{2} s_{1}^{\prime} s_{2}^{\prime}} \sum_{t_{1} t_{2} t_{1}^{\prime} t_{2}^{\prime}} j^{\mu}\left(1^{\prime}, 2^{\prime}, 1,2\right)_{A}^{*} j^{\nu}\left(1^{\prime}, 2^{\prime}, 1,2\right)_{A} .
\end{aligned}
$$

The two-body current matrix elements is antisymmetrized,

$$
j^{\mu}\left(1^{\prime}, 2^{\prime}, 1,2\right)_{A} \equiv j^{\mu}\left(1^{\prime}, 2^{\prime}, 1,2\right)-j^{\mu}\left(1^{\prime}, 2^{\prime}, 2,1\right) .
$$

The factor 1/4 in Eq. (25) accounts for the antisymmetry of the two-body wave function with respect to exchange of momenta, spin, and isospin quantum numbers.

In this work, we use Eq. (23) to compute the five $2 \mathrm{p} 2 \mathrm{~h}$ inclusive response functions $R_{C C}, R_{C L}, R_{L L}, R_{T}, R_{T^{\prime}}$, for neutrino and antineutrino scattering. Integrating over $\mathbf{p}_{2}^{\prime}$ using the momentum delta function, these responses are given by

$$
\begin{aligned}
R_{2 \mathrm{p} 2 \mathrm{~h}}^{K}= & \frac{V}{(2 \pi)^{9}} \int d^{3} p_{1}^{\prime} d^{3} h_{1} d^{3} h_{2} \frac{\left(m_{N}^{*}\right)^{4}}{E_{1} E_{2} E_{1}^{\prime} E_{2}^{\prime}} \Theta\left(p_{1}^{\prime}, h_{1}\right) \\
& \times \Theta\left(p_{2}^{\prime}, h_{2}\right) r^{K}\left(\mathbf{p}_{1}^{\prime}, \mathbf{p}_{2}^{\prime}, \mathbf{h}_{1}, \mathbf{h}_{2}\right) \\
& \times \delta\left(E_{1}^{\prime}+E_{2}^{\prime}-E_{1}-E_{2}-\omega\right),
\end{aligned}
$$

where $\mathbf{p}_{2}^{\prime}=\mathbf{h}_{\mathbf{1}}+\mathbf{h}_{\mathbf{2}}+\mathbf{q}-\mathbf{p}_{\mathbf{1}}^{\prime}$. The five elementary response functions for a $2 \mathrm{p} 2 \mathrm{~h}$ excitation $r^{K}$ are defined in Eqs. (7)-(11), in terms of the elementary hadronic tensor $w^{\mu \nu}$, Eq. (25), for $K=C C, C L, L L, T, T^{\prime}$. Because of axial symmetry around the momentum transfer $\mathbf{q}$ (the $z$ axis), we can fix the azimuthal angle of the first particle, $\phi_{1}^{\prime}=0$ and multiply by $2 \pi$. Finally, the energy delta function allows us to integrate analytically over $p_{1}^{\prime}$, reducing Eq. (27) to 7dimensional integral, that we compute numerically [58].

\section{Electroweak meson-exchange currents}

In this work, we use the electroweak MEC model described by the nine Feynman diagrams depicted in Fig. 1. The two-body current matrix elements $j^{\mu}\left(1^{\prime}, 2^{\prime}, 1,2\right)$ corresponding to this model enter in the calculation of the elementary $2 \mathrm{p} 2 \mathrm{~h}$ hadronic tensor, Eq. (25). The different contributions have been taken from the pion weak production model of Ref. [59].

To obtain the MEC, we have started from the pion production currents given in Eq. (51) of Ref. [59]. and we have applied the Goldberger-Treiman relation $f_{\pi N N} / m_{\pi}=$ $g_{A} /\left(2 f_{\pi}\right)$, with $f_{\pi}=93 \mathrm{MeV}$, to factorize a common coupling constant $f_{\pi N N} / m_{\pi}$ in all vertices $\pi N N$. We have not included the nucleon-pole diagrams where the $W^{ \pm}$boson is coupled to the nucleon current, since these diagrams are not considered part of the MEC and are not taken into account here. Some previous calculations of the correlations in the $2 \mathrm{p} 2 \mathrm{~h}$ channel, of pionic type [60] or of Jastrow type [61], indicate that their effect when added to the one-body response is to produce a tail to the right of the Quasielastic (QE) peak of the order of $10 \%$ of the total height.

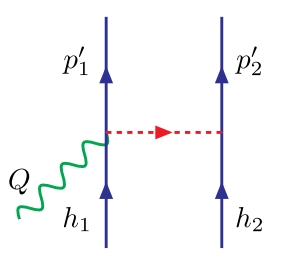

(a)

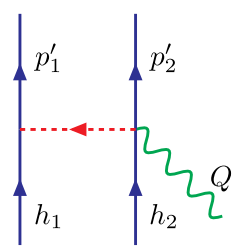

(b)

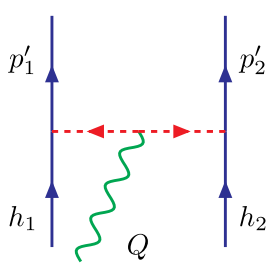

(c)

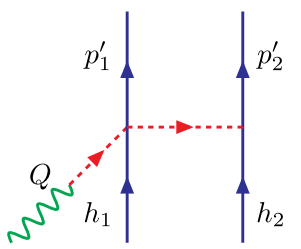

(d)

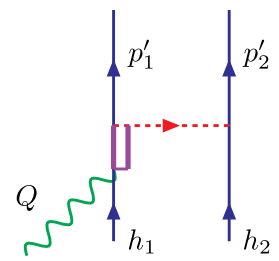

(f)

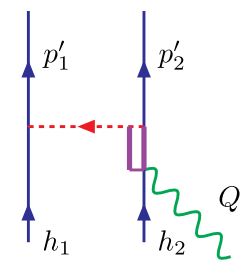

(g)

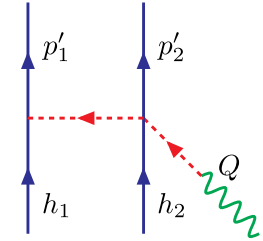

(e)

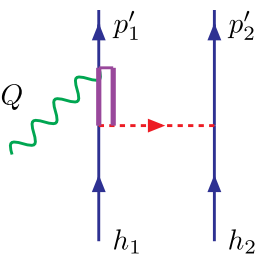

(h)

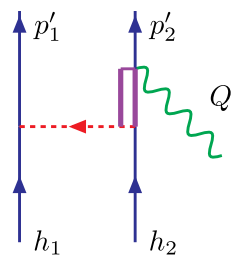

(i)

FIG. 1. Feynman diagrams for the electroweak MEC model used in this work. 
The MEC is the sum of four two-body currents: seagull (diagrams a and b), pion in flight (diagram c), pion-pole (diagrams $\mathrm{d}$ and $\mathrm{e}$ ), and $\Delta(1232)$ excitation forward (diagrams $\mathrm{f}$ and $\mathrm{g}$ ), and backward (diagrams $\mathrm{h}$ and $\mathrm{i}$ ).

$$
\begin{gathered}
j_{\text {sea }}^{\mu}=\left[I_{V}^{ \pm}\right]_{1^{\prime} 2^{\prime}, 12} \frac{f_{\pi N N}^{2}}{m_{\pi}^{2}} V_{\pi N N}^{s_{1}^{\prime} s_{1}}\left(\mathbf{p}_{1}^{\prime}, \mathbf{h}_{1}\right) F_{\pi N N}\left(k_{1}^{2}\right) \bar{u}_{s_{2}^{\prime}}\left(\mathbf{p}_{2}^{\prime}\right)\left[F_{1}^{V}\left(Q^{2}\right) \gamma_{5} \gamma^{\mu}+\frac{F_{\rho}\left(k_{2}^{2}\right)}{g_{A}} \gamma^{\mu}\right] u_{s_{2}}\left(\mathbf{h}_{2}\right)+(1 \leftrightarrow 2) \\
j_{\pi}^{\mu}=\left[I_{V}^{ \pm}\right]_{1^{\prime} 2^{\prime}, 12} \frac{f_{\pi N N}^{2}}{m_{\pi}^{2}} F_{1}^{V}\left(Q^{2}\right) V_{\pi N N}^{s_{1}^{\prime} s_{1}}\left(\mathbf{p}_{1}^{\prime}, \mathbf{h}_{1}\right) V_{\pi N N}^{s_{2}^{\prime} s_{2}}\left(\mathbf{p}_{2}^{\prime}, \mathbf{h}_{2}\right)\left(k_{1}^{\mu}-k_{2}^{\mu}\right) \\
j_{\text {pole }}^{\mu}=\left[I_{V}^{ \pm}\right]_{1^{\prime} 2^{\prime}, 12} \frac{f_{\pi N N}^{2}}{m_{\pi}^{2}} \frac{F_{\rho}\left(k_{1}^{2}\right)}{g_{A}} F_{\pi N N}\left(k_{2}^{2}\right) \frac{Q^{\mu} \bar{u}_{s_{1}^{\prime}}\left(\mathbf{p}_{1}^{\prime}\right) Q u_{s_{1}}\left(\mathbf{h}_{1}\right)}{Q^{2}-m_{\pi}^{2}} V_{\pi N N}^{s_{2}^{\prime} s_{2}}\left(\mathbf{p}_{2}^{\prime}, \mathbf{h}_{2}\right)+(1 \leftrightarrow 2) \\
j_{\Delta F}^{\mu}=\left[U_{\mathrm{F}}^{ \pm}\right]_{1^{\prime} 2^{\prime}, 12} \frac{f^{*} f_{\pi N N}}{m_{\pi}^{2}} V_{\pi N N}^{s_{2}^{\prime} s_{2}}\left(\mathbf{p}_{2}^{\prime}, \mathbf{h}_{2}\right) F_{\pi N \Delta}\left(k_{2}^{2}\right) \bar{u}_{s_{1}^{\prime}}\left(\mathbf{p}_{1}^{\prime}\right) k_{2}^{\alpha} G_{\alpha \beta}\left(h_{1}+Q\right) \Gamma^{\beta \mu}(Q) u_{s_{1}}\left(\mathbf{h}_{1}\right)+(1 \leftrightarrow 2) . \\
j_{\Delta B}^{\mu}=\left[U_{\mathrm{B}}^{ \pm}\right]_{1^{\prime} 2^{\prime}, 12} \frac{f^{*} f_{\pi N N}}{m_{\pi}^{2}} V_{\pi N N}^{s_{2}^{\prime} s_{2}}\left(\mathbf{p}_{2}^{\prime}, \mathbf{h}_{2}\right) F_{\pi N \Delta}\left(k_{2}^{2}\right) \bar{u}_{s_{1}^{\prime}}\left(\mathbf{p}_{1}^{\prime}\right) k_{2}^{\beta} \hat{\Gamma}^{\mu \alpha}(Q) G_{\alpha \beta}\left(p_{1}^{\prime}-Q\right) u_{s_{1}}\left(\mathbf{h}_{1}\right)+(1 \leftrightarrow 2) .
\end{gathered}
$$

where $k_{i}^{\mu}=\left(p_{i}^{\prime}-h_{i}\right)^{\mu}$ is the 4-momentum transferred to the $i$ th nucleon. Note that the energy of each nucleon includes the vector energy, Eq. (21), but the vector energies of the initial and final nucleons cancel out when computing the components $k_{i}^{0}=E_{i}^{\prime}-E_{i}$.

In these equations, we have defined the following function:

$$
V_{\pi N N}^{s_{1}^{\prime} s_{1}}\left(\mathbf{p}_{1}^{\prime}, \mathbf{h}_{1}\right) \equiv F_{\pi N N}\left(k_{1}^{2}\right) \frac{\bar{u}_{s_{1}^{\prime}}\left(\mathbf{p}_{1}^{\prime}\right) \gamma_{5} \not k_{1} u_{s_{1}}\left(\mathbf{h}_{1}\right)}{k_{1}^{2}-m_{\pi}^{2}} .
$$

This function appears in all the currents, describing the propagation and emission (or absorption) of the exchanged pion, having a strong form factor $F_{\pi N N}$ given by $[62,63]$

$$
F_{\pi N N}\left(k_{1}^{2}\right)=\frac{\Lambda^{2}-m_{\pi}^{2}}{\Lambda^{2}-k_{1}^{2}}
$$

and $\Lambda=1300 \mathrm{MeV}$.

The charge dependence of the currents in the different $\mathrm{p}$ $\mathrm{n}$ channels is determined by the matrix elements of the isospin operators $I_{V}^{ \pm}, U_{\mathrm{F}}^{ \pm}$, and $U_{\mathrm{B}}^{ \pm}$, where the $+(-)$sign refers to neutrino (antineutrino) scattering. They are defined by

$$
\begin{gathered}
I_{V}^{ \pm}=\left(I_{V}\right)_{x} \pm i\left(I_{V}\right)_{y} \\
U_{F}^{ \pm}=\left(U_{F}\right)_{x} \pm i\left(U_{F}\right)_{y} \\
U_{B}^{ \pm}=\left(U_{B}\right)_{x} \pm i\left(U_{B}\right)_{y},
\end{gathered}
$$

where

$$
\begin{gathered}
\boldsymbol{I}_{V}=i[\tau(1) \times \tau(2)] \\
\left(U_{F}\right)_{j}=\sqrt{\frac{3}{2}} \sum_{i=1}^{3}\left(T_{i} T_{j}^{\dagger}\right) \otimes \tau_{i} \\
\left(U_{B}\right)_{j}=\sqrt{\frac{3}{2}} \sum_{i=1}^{3}\left(T_{j} T_{i}^{\dagger}\right) \otimes \tau_{i},
\end{gathered}
$$

where $\vec{T}$ is an isovector transition operator from isospin $\frac{3}{2}$ to $\frac{1}{2}$.

Additionally, each current contains coupling constants and electroweak form factors. The coupling constants are $f_{\pi N N}=1, g_{A}=1.26$, and $f^{*}=2.13$.

The electroweak form factors are $F_{1}^{V}=F_{1}^{p}-F_{1}^{n}$ in the seagull vector and pion-in flight currents, for which we use Galster's parametrization [64], and $F_{\rho}$ in the axial seagull and pion-pole currents, taken from Ref. [59]. In the case of the $\Delta$ current, we use the form factors $C_{3}^{V}$ and $C_{5}^{A}$, respectively, for the vector and axial parts of the current [59]. These appear in the $N \rightarrow \Delta$ transition vertex in the forward current

$$
\Gamma^{\beta \mu}(Q)=\frac{C_{3}^{V}}{m_{N}}\left(g^{\beta \mu} Q-Q^{\beta} \gamma^{\mu}\right) \gamma_{5}+C_{5}^{A} g^{\beta \mu}
$$

and for the backward current

$$
\hat{\Gamma}^{\mu \alpha}(Q)=\gamma^{0}\left[\Gamma^{\alpha \mu}(-Q)\right]^{\dagger} \gamma^{0} .
$$

The vector and axial form factors in the $\Delta$ current are from Ref. [59], 


$$
\begin{aligned}
C_{3}^{V}\left(Q^{2}\right) & =\frac{2.13}{\left(1-Q^{2} / M_{V}^{2}\right)^{2}} \frac{1}{1-\frac{Q^{2}}{4 M_{V}^{2}}}, \\
C_{5}^{A}\left(Q^{2}\right) & =\frac{1.2}{\left(1-Q^{2} / M_{A \Delta}^{2}\right)^{2}} \frac{1}{1-\frac{Q^{2}}{4 M_{A \Delta}^{2}}},
\end{aligned}
$$

with $M_{V}=0.84 \mathrm{GeV}$ and $M_{A \Delta}=1.05 \mathrm{GeV}$.

We use the $\pi N \Delta$ strong form factor of Ref. [65], given by

$$
F_{\pi N \Delta}\left(k_{2}^{2}\right)=\frac{\Lambda_{\Delta}^{2}}{\Lambda_{\Delta}^{2}-k_{2}^{2}},
$$

where $\Lambda_{\Delta}=1150 \mathrm{MeV}$.

Finally, the $\Delta$-propagator, including the decay width of the $\Delta(1232)$, is given by

$$
G_{\alpha \beta}(P)=\frac{\mathcal{P}_{\alpha \beta}(P)}{P^{2}-M_{\Delta}^{2}+i M_{\Delta} \Gamma_{\Delta}\left(P^{2}\right)+\frac{\Gamma_{\Delta}\left(P^{2}\right)^{2}}{4}} .
$$

The projector $\mathcal{P}_{\alpha \beta}(P)$ over spin- $\frac{3}{2}$ on-shell particles is given by

$$
\begin{aligned}
\mathcal{P}_{\alpha \beta}(P)= & -\left(P+M_{\Delta}\right) \\
& \times\left[g_{\alpha \beta}-\frac{1}{3} \gamma_{\alpha} \gamma_{\beta}-\frac{2}{3} \frac{P_{\alpha} P_{\beta}}{M_{\Delta}^{2}}+\frac{1}{3} \frac{P_{\alpha} \gamma_{\beta}-P_{\beta} \gamma_{\alpha}}{M_{\Delta}}\right] .
\end{aligned}
$$

This concludes the description of the electroweak current. The spinors of particles and holes are calculated in the RMF model with the relativistic effective mass $m_{N}^{*}$ instead of $m_{N}$, and with on-shell energy, Eq. (19). The total nucleon energy in the RMF (21) includes the vector energy, $E_{v}$. As can be seen, this vector energy cancels out in the terms of the currents that depend on the vectors $k_{i}^{\mu}$. But it is not canceled in the delta propagator, which is the only place where $E_{v}$ will appear explicitly.

Note that when considering the free $\Delta$ without selfenergy we are neglecting the interaction of the $\Delta$ in the medium. The case of the interacting Delta is summarized in Appendix A and is briefly discussed at the end of Sec. IV.

The seagull current has a vector and an axial part. The pionic current is pure vector, and the pion-pole current is pure axial. However, the pion-pole could be considered as the axial part of the pion in flight in a certain sense. The $\Delta$-forward and backward currents have a vector and an axial part also.

In the case of electron scattering, the above formulas are valid, but only the vector part of the current appears. Thus, the pion-pole diagrams (d and e) are not contributing. The isospin operators are modified to the $z$ component instead of the \pm component [53]. Only the $L$ and $T$ responses are present in $\left(e, e^{\prime}\right)$

The exact calculation of the response functions $R_{K}(q, \omega)$ with this model of currents is carried out by numerical integration in seven dimensions of Eq. (27). The spin sums are performed numerically. The isospin channels in the case of neutrino CC scattering are $p n$ and $p p$ in the final states. These two channels are computed separately and then added to obtain the total responses. More details on the calculation are given in Ref. [66].

Note that the same $2 \mathrm{p} 2 \mathrm{~h}$ responses were calculated in a previous work $[20,46]$ with a similar MEC model. The differences with our new model are in the description of the $\Delta$ propagator, which is included here in its entirety, while in Refs. [20,46], only the real part of the denominator of the $\Delta$ propagator was included. The second difference is that in the present model the nucleon wave functions are solutions of the Dirac equation in the mean field, that is, Dirac plane waves with relativistic effective mass and vector energy. On the contrary, in Refs. [20,46], a RFG without effective mass was used, but including an energy shift of $40 \mathrm{MeV}$. As seen in Ref. [53], the effect of the imaginary part of the propagator is to increase the contribution of the $\Delta F$ diagram and the position of the maximum. The effect of the effective mass and vector energy is to reduce the height of the peak, which partially compensates for the difference of the MEC with respect to the calculation of Refs. [20,46].

Finally, the present model is restricted to a neutrino energy range in which excitations of higher resonances, beyond the Delta, are not essential. This is good enough for experiments like T2K but not for DUNE.

\section{Phase-space integral}

Part of the dependence of the $2 \mathrm{p} 2 \mathrm{~h}$ response as a function of $q$ and $\omega$ will be associated with the number of $2 \mathrm{p} 2 \mathrm{~h}$ states that can be excited while conserving energy and momentum. That number is proportional to the phase-space function. In the RMF of nuclear matter, it is given by

$$
\begin{aligned}
F(q, \omega)= & \int d^{3} p_{1}^{\prime} d^{3} h_{1} d^{3} h_{2} \frac{\left(m_{N}^{*}\right)^{4}}{E_{1} E_{2} E_{1}^{\prime} E_{2}^{\prime}} \Theta\left(p_{1}^{\prime}, h_{1}\right) \\
& \times \Theta\left(p_{2}^{\prime}, h_{2}\right) \delta\left(E_{1}^{\prime}+E_{2}^{\prime}-E_{1}-E_{2}-\omega\right),
\end{aligned}
$$

where $\mathbf{p}_{\mathbf{2}}^{\prime}=\mathbf{h}_{\mathbf{1}}+\mathbf{h}_{\mathbf{2}}+\mathbf{q}-\mathbf{p}_{\mathbf{1}}^{\prime}$. This function was fully studied in Refs. $[58,67]$. The phase space determines the global behavior of the $2 \mathrm{p} 2 \mathrm{~h}$ responses, on top of the additional modifications introduced by the particular model of two-body current operator. The main modification of the phase-space behavior in the responses is produced by the $Q^{2}$ dependence of the electroweak form factors. The rest of the dependency on $(q, \omega)$ comes from the structure of the different Feynman diagrams, which are dominated by the forward $\Delta$ excitations.

In this work, we approximate the phase space using the frozen nucleon approximation [58,67], consisting in neglecting the momenta of the holes compared to the momentum transfer, which usually is larger than $k_{F}$. Setting $\mathbf{h}_{1}=\mathbf{h}_{2}=0$, and $E_{1}=E_{2}=m_{N}^{*}$ inside the integral (47), one can perform the integral over $\mathbf{h}_{1}, \mathbf{h}_{2}$, 


$$
\begin{aligned}
F(q, \omega)_{\text {frozen }}= & \left(\frac{4}{3} \pi k_{F}^{3}\right)^{2} \int d^{3} p_{1}^{\prime} \frac{\left(m_{N}^{*}\right)^{2}}{E_{1}^{\prime} E_{2}^{\prime}} \Theta\left(p_{1}^{\prime}, 0\right) \\
& \times \Theta\left(p_{2}^{\prime}, 0\right) \delta\left(E_{1}^{\prime}+E_{2}^{\prime}-2 m_{N}^{*}-\omega\right) .
\end{aligned}
$$

This integral can be reduced to one dimension, integrating over the momentum $p_{1}^{\prime}$ and the azimuthal angle $\phi_{1}^{\prime}$ of the first ejected nucleon. In Ref. [58], the phase-space function (47) was studied by computing the seven-dimensional integral numerically and compared to the frozen approximation (48). This approximation is very good even for low momenta, except in the very low-energy threshold zone, where the $2 \mathrm{p} 2 \mathrm{~h}$ cross section is very small.

If we neglect Pauli blocking, the integral in Eq. (48) can be made analytically by going to the center-of-mass frame of the two final nucleons [67]. The following approximate formula is obtained:

$$
\begin{aligned}
F(q, \omega) & \simeq F(q, \omega)_{\text {frozen }} \\
& \simeq 4 \pi\left(\frac{4}{3} \pi k_{F}^{3}\right)^{2} \frac{m_{N}^{* 2}}{2} \sqrt{1-\frac{4 m_{N}^{* 2}}{\left(2 m_{N}^{*}+\omega\right)^{2}-q^{2}}} .
\end{aligned}
$$

From this equation, we obtain the minimum $\omega$ to excite a $2 \mathrm{p} 2 \mathrm{~h}$ state for fixed $q$, in this approximation,

$$
\omega_{\min }=\sqrt{4 m_{N}^{* 2}+q^{2}}-2 m_{N}^{*} .
$$

The phase-space integral is zero below this value. Note that this is the kinetic energy of a particle with mass $2 m_{N}^{*}$ and momentum $q$.

\section{E. Averaged $\Delta$ propagator}

When a $\Delta$ is excited in the $\Delta$-forward diagrams (f) and (g) of Fig. 1, a broad resonant peak is produced. This produces the dominant contribution to the MEC responses in the region around the $\Delta$ peak. To get this resonant peak in the semiempirical formula, we will approximate it by an average of the $\Delta$ propagator over the Fermi gas.

The peak of the $2 \mathrm{p} 2 \mathrm{~h}$ responses is due to the denominator in the $\Delta$ propagator for the forward diagrams,

$$
G_{\Delta}(H+Q) \equiv \frac{1}{(H+Q)^{2}-M_{\Delta}^{2}+i M_{\Delta} \Gamma_{\Delta}+\frac{\Gamma_{\Delta}^{2}}{4}}
$$

where $H^{\mu}=\left(E_{\mathrm{RMF}}, \mathbf{h}\right)$ is the 4-momentum of the hole. The square of the momentum in the denominator can be written

$$
\begin{aligned}
(H+Q)^{2} & =\left(E_{\mathrm{RMF}}+\omega\right)^{2}-(\mathbf{h}+\mathbf{q})^{2} \\
& \simeq\left(m_{N}^{*}+E_{v}+\omega\right)^{2}-2 \mathbf{h} \cdot \mathbf{q}-q^{2},
\end{aligned}
$$

where the energy of the hole has been approximated by $E_{\mathrm{RMF}}=m_{N}^{*}+E_{v}$ in the RMF model, by neglecting the kinetic energy of the first particle, and the momentum $h^{2}$ has also been neglected compared to the momentum transfer $q^{2}$. In this nonrelativistic limit for the initial nucleon, an average of the propagator (51) can be computed analytically,

$$
\begin{aligned}
G_{\mathrm{av}}(Q)= & G_{\mathrm{av}}(q, \omega)=\frac{1}{\frac{4}{3} \pi k_{F}^{3}} \int \frac{d^{3} h \theta\left(k_{F}-|\mathbf{h}|\right)}{a-2 \mathbf{h} \cdot \mathbf{q}+i b}, \\
= & \frac{1}{\frac{4}{3} \pi k_{F}^{3}} \frac{\pi}{q}\left\{\frac{(a+i b) k_{F}}{2 q}+\frac{4 q^{2} k_{F}^{2}-(a+i b)^{2}}{8 q^{2}}\right. \\
& \left.\times \ln \left[\frac{a+2 k_{F} q+i b}{a-2 k_{F} q+i b}\right]\right\},
\end{aligned}
$$

where the functions $a$ and $b$ are defined by

$$
\begin{gathered}
a \equiv m_{N}^{* 2}+\left(\omega+E_{v}\right)^{2}-q^{2} \\
+2 m_{N}^{*}\left(\omega+E_{v}+\Sigma\right)-M_{\Delta}^{2}+\frac{\Gamma^{2}}{4} \\
b \equiv M_{\Delta} \Gamma .
\end{gathered}
$$

Note that we have included a shift parameter $\Sigma(q)$, to obtain the correct position of the smeared $\Delta$ peak. The $\Delta$ width $\Gamma_{\Delta}$ will be replaced by an effective width on the average $\Gamma(q)$.

The effective width and shift are used only in the averaged propagator. In the exact calculation of the MEC, there is no shift, and the well-known value of the width of the Delta $\Gamma_{\Delta}\left(Q^{2}\right)$ is used. In Ref. [41], it was shown that the averaged propagator (in Ref. [41], it was called the "frozen" propagator) describes the $\omega$ dependence of the $2 \mathrm{p} 2 \mathrm{~h}$ responses only if effective values of $\Gamma(q)$ and $\Sigma(q)$ are used. The effective values are taken as parameters in the semiempirical formula, and they are fitted to the exact responses. As explained in detail in Ref. [41], in the exact responses, the $\Delta$ propagator inside the sevendimensional integral is being multiplied by a $q$-dependent weight determined by the matrix elements of the MEC. This changes the position and width of the MEC peak with respect to the simple average of the denominator.

The effective shift and width parameters, $\Sigma(q)$, and $\Gamma(q)$, will be adjusted with the semiempirical formula of the next section, for each value of the momentum transfer $q$.

\section{SEMIEMPIRICAL FORMULAS OF MEC RESPONSES}

In this section, we propose the semiempirical formulas for the nuclear responses, $R_{K}(q, \omega)$, by separating the contributions of the different Feynman diagrams of MEC and the interferences between them and extracting the contribution from the phase space, electroweak form factors, and coupling constants, corresponding to each term of the current. In the case of the $\Delta$-forward current, we also extract an average value of the $\Delta$ propagator. Much 
of the dependence on $q$ and $\omega$ is coming from these factors. The remaining dependence is coded into coefficients $\tilde{C}_{i}(q)$ that are assumed to only depend on $q$.

First, we write the MEC as

$$
j^{\mu}=j_{\mathrm{SP}}^{\mu}+j_{\Delta F}^{\mu}+j_{\Delta B}^{\mu},
$$

where the seagull-pionic (SP) contribution is the sum of Figs. 1(a)-1(e),

$$
j_{\mathrm{SP}}^{\mu}=j_{\text {sea }}^{\mu}+j_{\pi}^{\mu}+j_{\text {pole }}^{\mu} .
$$

Now, to compute the hadronic tensor $w^{\mu \nu}$, Eq. (25), we deal with products of the kind

$$
\begin{aligned}
j^{\mu *} j^{\nu}= & j_{\mathrm{SP}}^{\mu *} j_{\mathrm{SP}}^{\nu}+j_{\Delta F}^{\mu *} j_{\Delta F}^{\nu}+j_{\Delta B}^{\mu *} j_{\Delta B}^{\nu}+j_{S P}^{\mu *} j_{\Delta F}^{\nu}+j_{\Delta F}^{\mu *} j_{S P}^{\nu} \\
& +j_{\mathrm{SP}}^{\mu *} j_{\Delta B}^{\nu}+j_{\Delta B}^{\mu *} j_{\mathrm{SP}}^{\nu}+j_{\Delta F}^{\mu *} j_{\Delta B}^{\nu}+j_{\Delta B}^{\mu *} j_{\Delta F}^{\nu} .
\end{aligned}
$$

Using this expansion, the response functions (7)-(11) can be written as the sum of six subresponses corresponding to SP, $\Delta F$, and $\Delta B$, plus the interferences $\Delta F-\mathrm{SP}, \Delta B-\mathrm{SP}$, and $\Delta F-\Delta B$,

$$
\begin{aligned}
R^{K}(q, \omega)= & R_{\mathrm{SP}}^{K}+R_{\Delta F}^{K}+R_{\Delta B}^{K}+R_{\Delta F-\mathrm{SP}}^{K} \\
& +R_{\Delta B-\mathrm{SP}}^{K}+R_{\Delta F-\Delta B}^{K} ;
\end{aligned}
$$

this is a general expansion for all responses. In the case of the $C C, C L, L L$, and $T$ responses, we can also separate the contribution of the vector and axial parts of the current,

$$
R^{K}(q, \omega)=R^{K, V V}+R^{K, A A}, \quad K=C C, C L, L L, T,
$$

and their expansion is

$$
\begin{aligned}
R^{K, V V}(q, \omega)= & R_{\mathrm{SP}}^{K, V V}+R_{\Delta F}^{K, V V}+R_{\Delta B}^{K, V V}+R_{\Delta F-\mathrm{SP}}^{K, V V} \\
& +R_{\Delta B-\mathrm{SP}}^{K, V V}+R_{\Delta F-\Delta B}^{K, V V} \\
R^{K, A A}(q, \omega)= & R_{\mathrm{SP}}^{K, A A}+R_{\Delta F}^{K, A A}+R_{\Delta B}^{K, A A}+R_{\Delta F-\mathrm{SP}}^{K, A A} \\
& +R_{\Delta B-\mathrm{SP}}^{K, A A}+R_{\Delta F-\Delta B}^{K, A A} .
\end{aligned}
$$

In the case of the $T^{\prime}$ response, only the vector-axial product contributes,

$$
\begin{aligned}
R^{T^{\prime}}(q, \omega)= & R^{T^{\prime}, V A}(q, \omega) \\
= & R_{\mathrm{SP}}^{T^{\prime}, V A}+R_{\Delta F}^{T^{\prime}, V A}+R_{\Delta B}^{T^{\prime}, V A} \\
& +R_{\Delta F-\mathrm{SP}}^{T^{\prime}, V A}+R_{\Delta B-\mathrm{SP}}^{T^{\prime}, V A}+R_{\Delta F-\Delta B}^{T^{\prime}, V A} .
\end{aligned}
$$

We have written the $2 \mathrm{p} 2 \mathrm{~h}$ response functions as sums of the subresponses vector-vector, $R_{I, J}^{K, V V}$; axial-axial, $R_{I, J}^{K, A A}$; and vector-axial, $R_{I, J}^{T^{\prime}, V A}$, with $I, J=\mathrm{SP}, \Delta F, \Delta B$. From each one of these subresponses, we factorize the electroweak form factors, the coupling constants, an average delta propagator $G_{\mathrm{av}}(q, \omega)$ for each $\Delta F$ current, and finally the phase space $\frac{V}{(2 \pi)^{9}} F(q, \omega)$. Then, for each subresponse, we propose a semiempirical formula. Schematically, the general structure will be

$$
\begin{aligned}
R_{i}(q, \omega)= & {[\text { phase }- \text { space }] \times[\text { coupling constants }] } \\
& \times[\text { form factors }] \times[\text { averaged } \Delta \text { propagators }] \\
& \times \tilde{C}_{i}(q)
\end{aligned}
$$

where we assume that the adjustable coefficients $\tilde{C}_{i}(q)$ do not depend on $\omega$, but only depend on $q$. This is the main hypothesis on which the parametrization is based, that is, that most of the $\omega$ dependence comes from phase space, form factors, and the averaged $\Delta$ propagator. This is justified a posteriori in the next section when we check the quality of the fit by comparison with the exact results. These coefficients will be fitted to the corresponding subresponses in an exact calculation. The coefficients can be interpreted as nuclear mean values of spin-isospin contributions of the Feynman diagrams for each subresponse in a $2 \mathrm{p} 2 \mathrm{~h}$ excitation.

Below, we write down the explicit formula for the 54 subresponses, taking into account that some of them may need two coefficients or some additional correction, which will be discussed in the next section.

\section{A. Response $R_{T}^{V V}$}

$$
\begin{gathered}
R_{\Delta F}^{T, V V}=\frac{V}{(2 \pi)^{9}} F(q, \omega)\left(\frac{f^{*} f_{\pi N N}}{m_{\pi}^{2}}\right)^{2}\left(\frac{C_{3}^{V}}{m_{N}}\right)^{2}\left[\tilde{C}_{1, V 1}\left(\operatorname{Re}\left(G_{\mathrm{av}}^{V}\right)\right)^{2}+\tilde{C}_{1, V 2}\left(\operatorname{Im}\left(G_{\mathrm{av}}^{V}\right)\right)^{2}\right]\left(m_{N}^{4}\right) \\
R_{\mathrm{SP}}^{T, V V}=\frac{V}{(2 \pi)^{9}} F(q, \omega)\left(\frac{f_{\pi N N}^{2}}{m_{\pi}^{2}}\right)^{2}\left(F_{1}^{V}\right)^{2}\left(\tilde{C}_{2, V} \cdot m_{N}^{-2}\right)\left[1-\frac{\omega-0.7 q}{m_{N}}\right]^{2} \\
R_{\Delta B}^{T, V V}=\frac{V}{(2 \pi)^{9}} F(q, \omega)\left(\frac{f^{*} f_{\pi N N}}{m_{\pi}^{2}}\right)^{2}\left(\frac{C_{3}^{V}}{m_{N}}\right)^{2}\left(\tilde{C}_{3, V}\right)
\end{gathered}
$$




$$
\begin{gathered}
R_{\Delta F-\mathrm{SP}}^{T, V V}=\frac{V}{(2 \pi)^{9}} F(q, \omega)\left(\frac{f^{*} f_{\pi N N}^{3}}{m_{\pi}^{4}}\right)\left(\frac{C_{3}^{V}}{m_{N}}\right)\left(F_{1}^{V}\right)\left[\tilde{C}_{4, V 1}\left(\operatorname{Re}\left(G_{\mathrm{av}}^{V}\right)\right)+\tilde{C}_{4, V 2}\left(\operatorname{Im}\left(G_{\mathrm{av}}^{V}\right)\right)\right]\left(m_{N}\right) \\
R_{\Delta F-\Delta B}^{T, V V}=\frac{V}{(2 \pi)^{9}} F(q, \omega)\left(\frac{f^{*} f_{\pi N N}}{m_{\pi}^{2}}\right)^{2}\left(\frac{C_{3}^{V}}{m_{N}}\right)^{2}\left[\tilde{C}_{5, V 1}\left(\operatorname{Re}\left(G_{\mathrm{av}}^{V}\right)\right)+\tilde{C}_{5, V 2}\left(\operatorname{Im}\left(G_{\mathrm{av}}^{V}\right)\right)\right]\left(m_{N}^{2}\right) \\
R_{\Delta B-\mathrm{SP}}^{T, V V}=\frac{V}{(2 \pi)^{9}} F(q, \omega)\left(\frac{f^{*} f_{\pi N N}^{3}}{m_{\pi}^{4}}\right)\left(\frac{C_{3}^{V}}{m_{N}}\right)\left(F_{1}^{V}\right)\left(\tilde{C}_{6, V} \cdot m_{N}^{-1}\right)
\end{gathered}
$$

\section{B. Response $R_{T}^{A A}$}

$$
\begin{gathered}
R_{\Delta F}^{T, A A}=\frac{V}{(2 \pi)^{9}} F(q, \omega)\left(\frac{f^{*} f_{\pi N N}}{m_{\pi}^{2}}\right)^{2}\left(C_{A}^{5}\right)^{2}\left[\tilde{C}_{1, A 1}\left(\operatorname{Re}\left(G_{\mathrm{av}}^{A}\right)\right)^{2}+\tilde{C}_{1, A 2}\left(\operatorname{Im}\left(G_{\mathrm{av}}^{A}\right)\right)^{2}\right]\left(m_{N}^{2}\right) \\
R_{\mathrm{SP}}^{T, A A}=\frac{V}{(2 \pi)^{9}} F(q, \omega)\left(\frac{f_{\pi N N}^{2}}{m_{\pi}^{2}}\right)^{2}\left(\frac{1}{g_{A}}\right)^{2}\left(\tilde{C}_{2, A} \cdot m_{N}^{-2}\right) \\
R_{\Delta F-\mathrm{SP}}^{T, A A}=\frac{V}{(2 \pi)^{9}} F(q, \omega)\left(\frac{f^{*} f_{\pi N N}^{3}}{m_{\pi}^{4}}\right)\left(C_{5}^{A}\right)\left(\frac{1}{g_{A}}\right)\left[\tilde{C}_{4, A 1}\left(\operatorname{Re}\left(G_{\mathrm{av}}^{A}\right)\right)+\tilde{C}_{4, A 2}\left(\operatorname{Im}\left(G_{\mathrm{av}}^{A}\right)\right)\right] \\
R_{\Delta F-\Delta B}^{T, A A}=\frac{V(q, \omega)\left(\frac{f^{*} f_{\pi N N}}{m_{\pi}^{2}}\right)^{2}\left(C_{5}^{A}\right)^{2}\left(\tilde{C}_{3, A} \cdot m_{N}^{-2}\right)}{(2 \pi)^{9}} F(q, \omega)\left(\frac{f^{*} f_{\pi N N}}{m_{\pi}^{2}}\right)^{2}\left(C_{5}^{A}\right)^{2}\left[\tilde{C}_{5, A 1}\left(\operatorname{Re}\left(G_{\mathrm{av}}^{A}\right)\right)+\tilde{C}_{5, A 2}\left(\operatorname{Im}\left(G_{\mathrm{av}}^{A}\right)\right)\right] \\
R_{\Delta B-\mathrm{SP}}^{T, A A}=\frac{V}{(2 \pi)^{9}} F(q, \omega)\left(\frac{f^{*} f_{\pi N N}^{3}}{m_{\pi}^{4}}\right)\left(C_{5}^{A}\right)\left(\frac{1}{g_{A}}\right)\left(\tilde{C}_{6, A} \cdot m_{N}^{-2}\right)
\end{gathered}
$$

\section{Response $R_{T^{\prime}}^{V A}$}

$$
\begin{aligned}
& R_{\Delta F}^{T^{\prime}, V A}=\frac{V}{(2 \pi)^{9}} F(q, \omega)\left(\frac{f^{*} f_{\pi N N}}{m_{\pi}^{2}}\right)^{2}\left(\frac{C_{3}^{V}}{m_{N}}\right)\left(C_{5}^{A}\right)\left[\tilde{C}_{1, V A 1}\left(\operatorname{Re}\left(G_{\mathrm{av}}^{V A}\right)\right)^{2}+\tilde{C}_{1, V A 2}\left(\operatorname{Im}\left(G_{\mathrm{av}}^{V A}\right)\right)^{2}\right]\left(m_{N}^{3}\right) \\
& R_{\mathrm{SP}}^{T^{\prime}, V A}=\frac{V}{(2 \pi)^{9}} F(q, \omega)\left(\frac{f_{\pi N N}^{2}}{m_{\pi}^{2}}\right)^{2}\left(F_{1}^{V}\right)\left(\frac{1}{g_{A}}\right)\left(\tilde{C}_{2, V A} \cdot m_{N}^{-2}\right)\left[1-\frac{\omega-0.7 q}{m_{N}}\right]^{2} \\
& R_{\Delta B}^{T^{\prime}, V A}=\frac{V}{(2 \pi)^{9}} F(q, \omega)\left(\frac{f^{*} f_{\pi N N}}{m_{\pi}^{2}}\right)^{2}\left(\frac{C_{3}^{V}}{m_{N}}\right)\left(C_{5}^{A}\right)\left(\tilde{C}_{3, V A} \cdot m_{N}^{-1}\right) \\
& R_{\Delta F-\mathrm{SP}}^{T^{\prime}, V A}=\frac{V}{(2 \pi)^{9}} F(q, \omega)\left(\frac{f^{*} f_{\pi N N}^{3}}{m_{\pi}^{4}}\right)\left|G_{\mathrm{av}}^{V A}\right|\left[\left(\frac{C_{3}^{V}}{m_{N}}\right)\left(\frac{1}{g_{A}}\right)\left(\tilde{C}_{4, V A 1} \cdot m_{N}\right)+\left(C_{5}^{A}\right)\left(F_{1}^{V}\right)\left(\tilde{C}_{4, V A 2}\right)\right] \\
& R_{\Delta F-\Delta B}^{T^{\prime}, V A}=\frac{V}{(2 \pi)^{9}} F(q, \omega)\left(\frac{f^{*} f_{\pi N N}}{m_{\pi}^{2}}\right)^{2}\left(\frac{C_{3}^{V}}{m_{N}}\right)\left(C_{5}^{A}\right)\left[\tilde{C}_{5, V A 1}\left(\operatorname{Re}\left(G_{\mathrm{av}}^{V A}\right)\right)+\tilde{C}_{5, V A 2}\left(\operatorname{Im}\left(G_{\mathrm{av}}^{V A}\right)\right)\right] 2 m_{N} \\
& R_{\Delta B-\mathrm{SP}}^{T^{\prime}, V A}=\frac{V}{(2 \pi)^{9}} F(q, \omega)\left(\frac{f^{*} f_{\pi N N}^{3}}{m_{\pi}^{4}}\right)\left[\left(\frac{C_{3}^{V}}{m_{N}}\right)\left(\frac{1}{g_{A}}\right)\left(\tilde{C}_{6, V A} \cdot m_{N}^{-1}\right)+\left(C_{5}^{A}\right)\left(F_{1}^{V}\right)\left(\tilde{C}_{6, A V} \cdot m_{N}^{-2}\right)\right]
\end{aligned}
$$




\section{Response $R_{C C}^{V V}$}

$$
\begin{gathered}
R_{\Delta F}^{C C, V V}=\frac{V}{(2 \pi)^{9}} F(q, \omega)\left(\frac{f^{*} f_{\pi N N}}{m_{\pi}^{2}}\right)^{2}\left(\frac{C_{3}^{V}}{m_{N}}\right)^{2}\left[\tilde{C}_{1, V 1}\left(\operatorname{Re}\left(G_{\mathrm{av}}^{V}\right)\right)^{2}+\tilde{C}_{1, V 2}\left(\operatorname{Im}\left(G_{\mathrm{av}}^{V}\right)\right)^{2}\right]\left(m_{N}^{4}\right) \\
R_{S P}^{C C, V V}=\frac{V}{(2 \pi)^{9}} F(q, \omega)\left(\frac{f_{\pi N N}^{2}}{m_{\pi}^{2}}\right)^{2}\left(F_{1}^{V}\right)^{2}\left(\tilde{C}_{2, V} \cdot m_{N}^{-2}\right) \\
R_{\Delta B}^{C C, V V}=\frac{V}{(2 \pi)^{9}} F(q, \omega)\left(\frac{f^{*} f_{\pi N N}}{m_{\pi}^{2}}\right)^{2}\left(\frac{C_{3}^{V}}{m_{N}}\right)^{2}\left(\tilde{C}_{3, V}\right) \\
R_{\Delta F-\mathrm{SP}}^{C C, V}=\frac{V}{(2 \pi)^{9}} F(q, \omega)\left(\frac{f^{*} f_{\pi N N}^{3}}{m_{\pi}^{4}}\right)\left(\frac{C_{3}^{V}}{m_{N}}\right)\left(F_{1}^{V}\right)\left[\tilde{C}_{4, V 1}\left(\operatorname{Re}\left(G_{\mathrm{av}}^{V}\right)\right)+\tilde{C}_{4, V 2}\left(\operatorname{Im}\left(G_{\mathrm{av}}^{V}\right)\right)\right]\left(m_{N}\right) \\
R_{\Delta F-\Delta B}^{C C, V V}=\frac{V}{(2 \pi)^{9}} F(q, \omega)\left(\frac{f^{*} f_{\pi N N}}{m_{\pi}^{2}}\right)^{2}\left(\frac{C_{3}^{V}}{m_{N}}\right)^{2}\left[\tilde{C}_{5, V 1}\left(\operatorname{Re}\left(G_{\mathrm{av}}^{V}\right)\right)+\tilde{C}_{5, V 2}\left(\operatorname{Im}\left(G_{\mathrm{av}}^{V}\right)\right)\right]\left(m_{N}^{2}\right) \\
R_{\Delta B-S P}^{C C, V V}=\frac{V}{(2 \pi)^{9}} F(q, \omega)\left(\frac{f^{*} f_{\pi N N}^{3}}{m_{\pi}^{4}}\right)\left(\frac{C_{3}^{V}}{m_{N}}\right)\left(F_{1}^{V}\right)\left(\tilde{C}_{6, V} \cdot m_{N}^{-1}\right)
\end{gathered}
$$

\section{E. Response $R_{C C}^{A A}$}

$$
\begin{gathered}
R_{\Delta F}^{C C, A A}=\frac{V}{(2 \pi)^{9}} F(q, \omega)\left(\frac{f^{*} f_{\pi N N}}{m_{\pi}^{2}}\right)^{2}\left(C_{A}^{5}\right)^{2}\left[\tilde{C}_{1, A 1}\left(\operatorname{Re}\left(G_{\mathrm{av}}^{A}\right)\right)^{2}+\tilde{C}_{1, A 2}\left(\operatorname{Im}\left(G_{\mathrm{av}}^{A}\right)\right)^{2}\right]\left(m_{N}^{2}\right) \\
R_{\mathrm{SP}}^{C C, A A}=\frac{V}{(2 \pi)^{9}} F(q, \omega)\left(\frac{f_{\pi N N}^{2}}{m_{\pi}^{2}}\right)^{2}\left(\frac{1}{g_{A}}\right)^{2}\left[\tilde{C}_{2, A 1}+\tilde{C}_{2, A 2}\left(\frac{\omega \cdot m_{N}}{Q^{2}-m_{\pi}^{2}}\right)^{2}\right]\left(m_{N}^{-2}\right) \\
R_{\Delta B}^{C C, A A}=\frac{V}{(2 \pi)^{9}} F(q, \omega)\left(\frac{f^{*} f_{\pi N N}}{m_{\pi}^{2}}\right)^{2}\left(C_{5}^{A}\right)^{2}\left(\tilde{C}_{3, A} \cdot m_{N}^{-2}\right) \\
R_{\Delta F-\mathrm{SP}}^{C C, A A}=\frac{V}{(2 \pi)^{9}} F(q, \omega)\left(\frac{f^{*} f_{\pi N N}^{3}}{m_{\pi}^{4}}\right)\left(C_{5}^{A}\right)\left(\frac{1}{g_{A}}\right)\left[\tilde{C}_{4, A 1}\left(\operatorname{Re}\left(G_{\mathrm{av}}^{A}\right)\right)+\tilde{C}_{4, A 2}\left(\operatorname{Im}\left(G_{\mathrm{av}}^{A}\right)\right)\right] \\
R_{\Delta F-\Delta B}^{C C, A A}=\frac{V}{(2 \pi)^{9}} F(q, \omega)\left(\frac{f^{*} f_{\pi N N}}{m_{\pi}^{2}}\right)^{2}\left(C_{5}^{A}\right)^{2}\left[\tilde{C}_{5, A 1}\left(\operatorname{Re}\left(G_{\mathrm{av}}^{A}\right)\right)+\tilde{C}_{5, A 2}\left(\operatorname{Im}\left(G_{\mathrm{av}}^{A}\right)\right)\right] \\
R_{\Delta B-\mathrm{SP}}^{C C, A A}=\frac{V}{(2 \pi)^{9}} F(q, \omega)\left(\frac{f^{*} f_{\pi N N}^{3}}{m_{\pi}^{4}}\right)\left(C_{5}^{A}\right)\left(\frac{1}{g_{A}}\right)\left(\tilde{C}_{6, A} \cdot m_{N}^{-2}\right)
\end{gathered}
$$

\section{F. Responses $R_{C L}^{V V}$ and $R_{L L}^{V V}$}

These responses are computed assuming conservation of the vector current:

$$
\begin{aligned}
& R^{C L, V V}=-\frac{\omega}{q} R^{C C, V V} \\
& R^{L L, V V}=\frac{\omega^{2}}{q^{2}} R^{C C, V V}
\end{aligned}
$$




\section{G. Responses $\boldsymbol{R}_{C L}^{A A}$ and $\boldsymbol{R}_{L L}^{A A}$}

The semiempirical formulas for $R_{C L}^{A A}$ and $R_{L L}^{A A}$ are similar to the $R_{C C}^{A A}$. Only the numerical values of the coefficients $\tilde{C}_{i}$ change.

\section{H. Electromagnetic responses $\boldsymbol{R}_{e m}^{L}$ and $\boldsymbol{R}_{e m}^{T}$}

It can be shown with the formalism of Ref. [66] that for symmetric nuclear matter the electromagnetic $2 \mathrm{p} 2 \mathrm{~h}$ responses are one-half of the $V V$ weak responses

$$
\begin{aligned}
R_{e m}^{L} & =\frac{1}{2} R^{C C, V V} \\
R_{e m}^{T} & =\frac{1}{2} R^{T, V V} .
\end{aligned}
$$

Therefore, the same semiempirical formulas for the VV responses apply for the electromagnetic responses with a factor $1 / 2$.

\section{Properties of semiempirical formulas}

Here, we describe and clarify some particularities about the semiempirical formulas (66)-(95) for the subresponses and their theoretical meaning:

(i) All the dependence on $\omega$ is analytical. So, the semiempirical expansion assumes that the $\omega$ dependence comes mainly from the product of phase space $F(q, \omega)$, electroweak form factors, and averaged $\Delta$ propagator. The only exceptions are $R_{\mathrm{SP}}^{T, V V}$ and $R_{\mathrm{SP}}^{T^{\prime}, V A}$ subresponses, which include an $\omega$-dependent factor that is obtained empirically by comparing with the exact result.

(ii) The coefficients $\tilde{C}_{i}$ in all the formulas are dimensionless. That is why powers of nucleon masses have been introduced in the subresponses.

(iii) The phase space $F(q, \omega)$ is common to all the formulas. In this work, it is computed analytically using the approximation (49).

(iv) All the responses are proportional to the volume $V=$ $(2 \pi)^{3} Z /\left(\frac{8}{3} \pi k_{F}^{3}\right)$ for symmetric nuclear matter $Z=N$. For asymmetric matter, the formulas should be modified using two different Fermi momenta for protons and neutrons.

(v) Each subresponse includes a specific product of form factors and coupling constants, except the axial SP subresponses, which do not allow us to extract explicitly the form factor $F_{\rho}\left(k_{i}\right)$.

(vi) In most subresponses, the averaged $\Delta$ propagator appears separated in real and imaginary parts, each one multiplied by a parameter $\tilde{C}_{i}$. The only exception is the $R_{\Delta F-S P}^{T^{\prime}, V A}$, which only include the modulus $\left|G_{\mathrm{av}}\right|$. Note that in the formulas there are three versions of the averaged propagator: $G_{\mathrm{av}}^{V}$ for the $V V$ responses, $G_{\mathrm{av}}^{A}$ for the $A A$ subresponses, and $G_{\mathrm{av}}^{V A}$ for the $T^{\prime}, \quad V A$ responses. They differ in the values of the effective width, $\Gamma$, and shift, $\Sigma$, of the $\Delta$ propagator. The corresponding six parameters are denoted by $\Gamma_{V}, \Gamma_{A}, \Gamma_{V A}, \Sigma_{V}, \Sigma_{A}$, and $\Sigma_{V A}$.

Finally, the semiempirical formulas allow us to calculate analytically and directly all the $2 \mathrm{p} 2 \mathrm{~h}$ electroweak responses. For a fixed value of $q$, the total $2 \mathrm{p} 2 \mathrm{~h}$ response depends on the sum of all the subresponses, with a total of 73 parameters. This number of parameters may seem large. Note that the axial part and the vector part of the five response functions, as well as their interferences, are described simultaneously. This could be compared with the parametrization of Refs. [20,46], which needs about 56 parameters to describe all the $2 \mathrm{p} 2 \mathrm{~h}$ responses in another nuclear model, based on the RFG and not RMF, and also using only the real part of the $\Delta$ propagator. Here, we use the full $\Delta$ propagator [53]. However, the present parametrization is an advance since we obtain explicit dependence of the responses on physical magnitudes-form factors, coupling constants, Fermi momentum, etc- that can be modified a posteriori if desired. Also, as we show below, many of these 54 subresponses are very small and could safely neglected, leaving us with a smaller number of parameters. However, in this paper, we have computed all the subresponses.

\section{RESULTS}

In this section, we obtain the values of the coefficients of the semiempirical formulas (66)-(95) and the parameters $\Gamma$ and $\Sigma$ in the averaged $\Delta$ propagator (54), (55), (56). Since the experimental responses are not available and it is not possible to obtain them phenomenologically, the coefficients cannot be obtained directly from data. Therefore, the only possibility is to fit a theoretical model. In our case, the interest is to obtain a parametrization of the responses to make theoretical predictions of neutrino cross sections in a computationally fast way. All numerical results in this section correspond to ${ }^{12} \mathrm{C}$ with $k_{F}=225 \mathrm{MeV} / \mathrm{c}$, $M^{*}=0.8$, and $E_{v}=141 \mathrm{MeV}$, fitted in Ref. [53].

First, we have calculated the "exact" subresponses for a set of kinematics, $(q, \omega)$, performing numerical integrations in seven dimensions in the RMF of nuclear matter described in Sec. II. Our computer code calculates the seven total $2 \mathrm{p} 2 \mathrm{~h}$ responses of neutrino CC scattering, $R_{K}$, $K=C C, C L, L L, T, T^{\prime}$, and electrons, $R_{\mathrm{em}}^{L}$ and $R_{\mathrm{em}}^{T}$, using Eq. (27). Our numerical code allows us to include in the calculation some specific Feynman diagrams and exclude others. To compute the subresponses, we have performed three runs with the individual currents, SP, $\Delta F$, and $\Delta B$, and three more runs with the pairs of currents $(\mathrm{SP}+\Delta F)$, $(\mathrm{SP}+\Delta B)$, and $(\Delta F+\Delta B)$. Subtracting the separate contributions of the single currents, the interference subresponses are obtained. For instance, 


$$
R_{\mathrm{SP}-\Delta F}=R_{\mathrm{SP}+\Delta F}-R_{\mathrm{SP}}-R_{\Delta F} .
$$

An additional run is performed with the full MEC to get the complete result. Each run requires computing the seven response functions in a grid of $(q, \omega)$ values, with $q=$ $200, \ldots, 2000 \mathrm{MeV} / \mathrm{c}$ in steps of $\Delta q=100$ and $\omega=$ $10, \ldots, q$ in steps of $\Delta \omega=10 \mathrm{MeV}$. The grid contains about 15,000 kinematical points $\left(q_{i}, \omega_{i}\right)$. Note that the computation for each kinematical point takes an average of 5 min on our high-performance processors. This means each run requires about 52 days in one processor and one year for the seven runs. We have used the PROTEUS scientific computing cloud of the ic1 [68] [2300 processors with total 90,000 Giga FLOP (floating point arithmetic operations)], allowing us to do the calculation in a few days.

Once we have stored the tables of the exact subresponses in the grid, we fit the coefficients of the semiempirical formula for fixed $q$. The fit is made by minimizing a $\chi^{2}$ function for each subresponse separately, thus obtaining the coefficients $\tilde{C}_{i}(q)$. The $R_{\Delta F}^{T, V V}$ responses are used to fit the effective width, $\Gamma_{V}(q)$, and shift, $\Sigma_{V}(q)$, of the averaged delta propagator as well. The same procedure is followed to fit the $A A$ and $V A$ effective widths and shifts using the responses $R_{\Delta F}^{T, A A}$, and $R_{\Delta F}^{T^{\prime}, V A}$, respectively. Therefore, in the transverse subresponses $\Delta F$, four coefficients are being adjusted simultaneously. Once the widths and shifts have been fitted in this way, they are set to that value in all the subresponses.

The coefficients obtained in the fit are tabulated in Appendix B. In Table I, the effective width, $\Gamma(q)$, and shift, $\Sigma(q)$, of the averaged $\Delta$ propagator, $G_{\text {av }}(q, \omega)$, are given for $V V, A A$, or $V A$ responses. The coefficients $\tilde{C}_{i}$ are provided in Tables II (for the $R_{T}^{V V}$ response function),
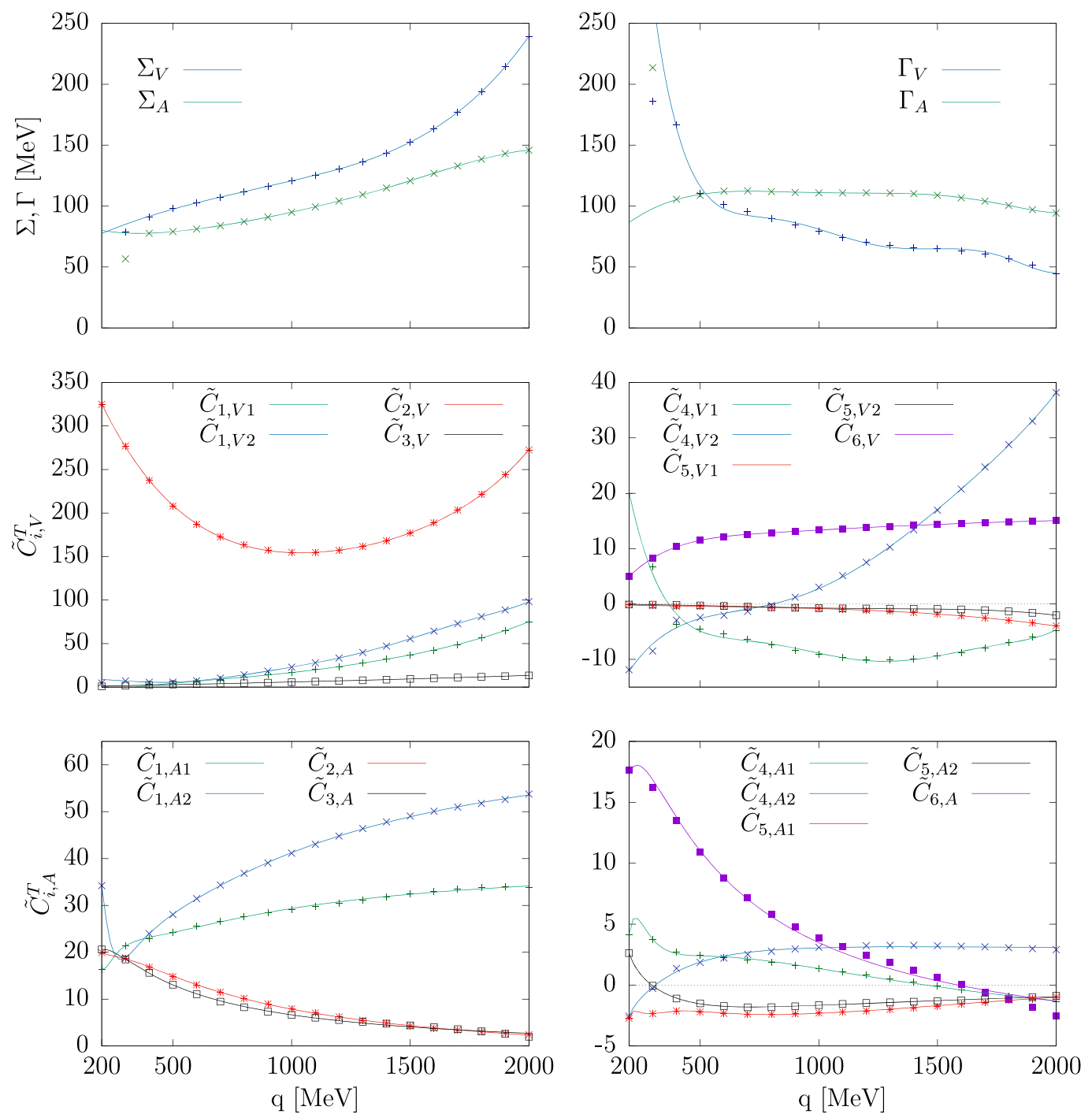

FIG. 2. Coefficients of the semiempirical formulas and parameters $\Gamma, \Sigma$ in the averaged $\Delta$ propagator, for the $R^{T} 2 \mathrm{p} 2 \mathrm{~h}$ response function plotted against the momentum transfer $q$. The dots are the fitted values, and the curves are the parametrizations with polynomial functions. Tables of these coefficients and parametrizations are given in the Appendix. 
III (for $R_{T}^{A A}$ ), IV (for $R_{C C}^{V V}$ ), V (for $R_{C C}^{A A}$ ), VI (for $R_{C L}^{A A}$ ), VII (for $R_{L L}^{A A}$ ), and VIII (for $R_{T^{\prime}}^{V A}$ ). The tables are given as a function of $q$ and can be interpolated for other $q$ values. We also provide polynomial parametrizations in Appendix C.

In Fig. 2, we plot the coefficients of the semiempirical formula for the case of the subresponses $R^{T}$ for $V V$ and $A A$ cases. We plot the coefficients $\tilde{C}_{i}$ and the effective widths, $\Gamma_{V}$ and $\Gamma_{A}$, and shifts, $\Sigma_{V}$ and $\Sigma_{A}$, of the averaged $\Delta$ propagator as a function of the momentum transfer $q$. Smooth dependence on $q$ is observed, except for very low $q$ below $300 \mathrm{MeV} / \mathrm{c}$ where the dependence is more abrupt in some cases, specifically in the case of the widths and shifts of the $\Delta$ propagator for $q<300 \mathrm{MeV} / \mathrm{c}$. The reason why there is an abrupt change for low momentum in the $\Delta$ current coefficients is because the peak of the delta is not reached below $q=300 \mathrm{MeV} / \mathrm{c}$, since at least the transferred energy must be large enough to produce the $\Delta$. In the present RMF model, this is $\omega=m_{\Delta}-m_{N}^{*}-E_{v} \simeq 340 \mathrm{MeV}$. Therefore, these coefficients are less restricted, and their value has greater indeterminacy when making the fit. Similar resultsnot shown in Fig. 2 - are obtained for the dependence on $q$ of the rest of the coefficients of the semiempirical formula.

The most relevant coefficients for calculating the responses are those corresponding to the $\Delta$-forward transverse responses ( $T$ and $T^{\prime}$ ) because these subresponses are dominant in the $\Delta$ region. These coefficients are $\tilde{C}_{1 V i}, \tilde{C}_{1 A i}$, and $\tilde{C}_{1 V A i}$. They increase moderately with $q$, and their values vary between $\tilde{C}_{i} \simeq 0$ and 100 for $200 \leq q \leq 2000 \mathrm{MeV} / \mathrm{c}$.
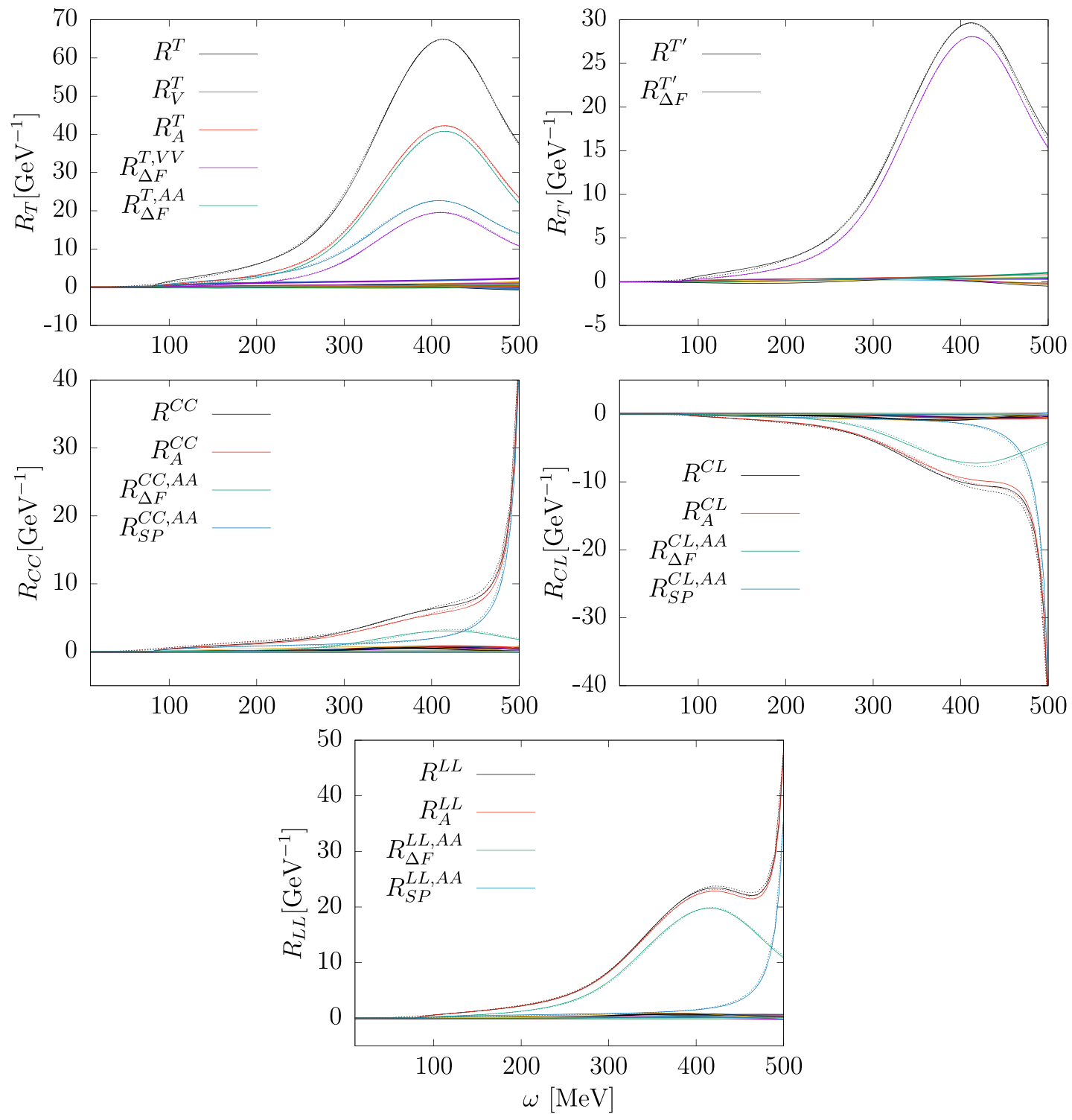

FIG. 3. Comparison between all the subresponses that contribute to each of the response functions for $q=500 \mathrm{MeV} / \mathrm{c}$. Only the most important subresponses appear in the legend. But they are all drawn. For each color, the solid line is the parametrization, and the dotted lines are the exact calculation. 
In Figs. 3 and 4, we show, as an example, all the responses and subresponses as a function of $\omega$ for two values of the momentum transfer $q=500$ and $1000 \mathrm{MeV} / \mathrm{c}$, respectively. The dominant subresponses in the responses $T$ and $T^{\prime}$ are the delta-forward ones, while the axial delta-forward and seagull-pionic response functions $C C, C L$, and $L L$. The rest of the subresponses give a very small contribution to the total and could in principle be neglected, although we have included all in the calculation. In the figures, we plot the exact result and semiempirical formula, using the parametrization of the $\tilde{C}_{i}$ parameters given in the Appendix $\mathrm{C}$, for each one of the dominant subresponses and also for the total responses.

In Fig. 5, it is seen that the total responses are well described by the semiempirical formula in the range of $q=$ $200, \ldots, 2000 \mathrm{MeV} / \mathrm{c}$ considered in the present work. The five $2 \mathrm{p} 2 \mathrm{~h}$ response functions for $\mathrm{CC}$ neutrino scattering from ${ }^{12} \mathrm{C}$, computed with the RMF model and with the semiempirical formula, are mostly identical in the scale of the figure. This indicates that the semiempirical formula can be used with guarantees to calculate the cross section in the $2 \mathrm{p} 2 \mathrm{~h}$ channel. For other values of $q$, it is enough to interpolate the tables of the coefficients of Appendix B, or to use the polynomial parametrizations of Appendix C. In Fig. 6, we give an example of how the formula works in the case of $\left(e, e^{\prime}\right)$ cross section of ${ }^{12} \mathrm{C}$ for various kinematics. The electron energy and the scattering angle are fixed in the experiment. When changing omega, the momentum transfer is not constant but depends on the three variables continuously. Then, it is necessary to interpolate the coefficients of the semiempirical formula to calculate the $2 \mathrm{p} 2 \mathrm{~h}$ cross section. In the figure, we used polynomial interpolation.
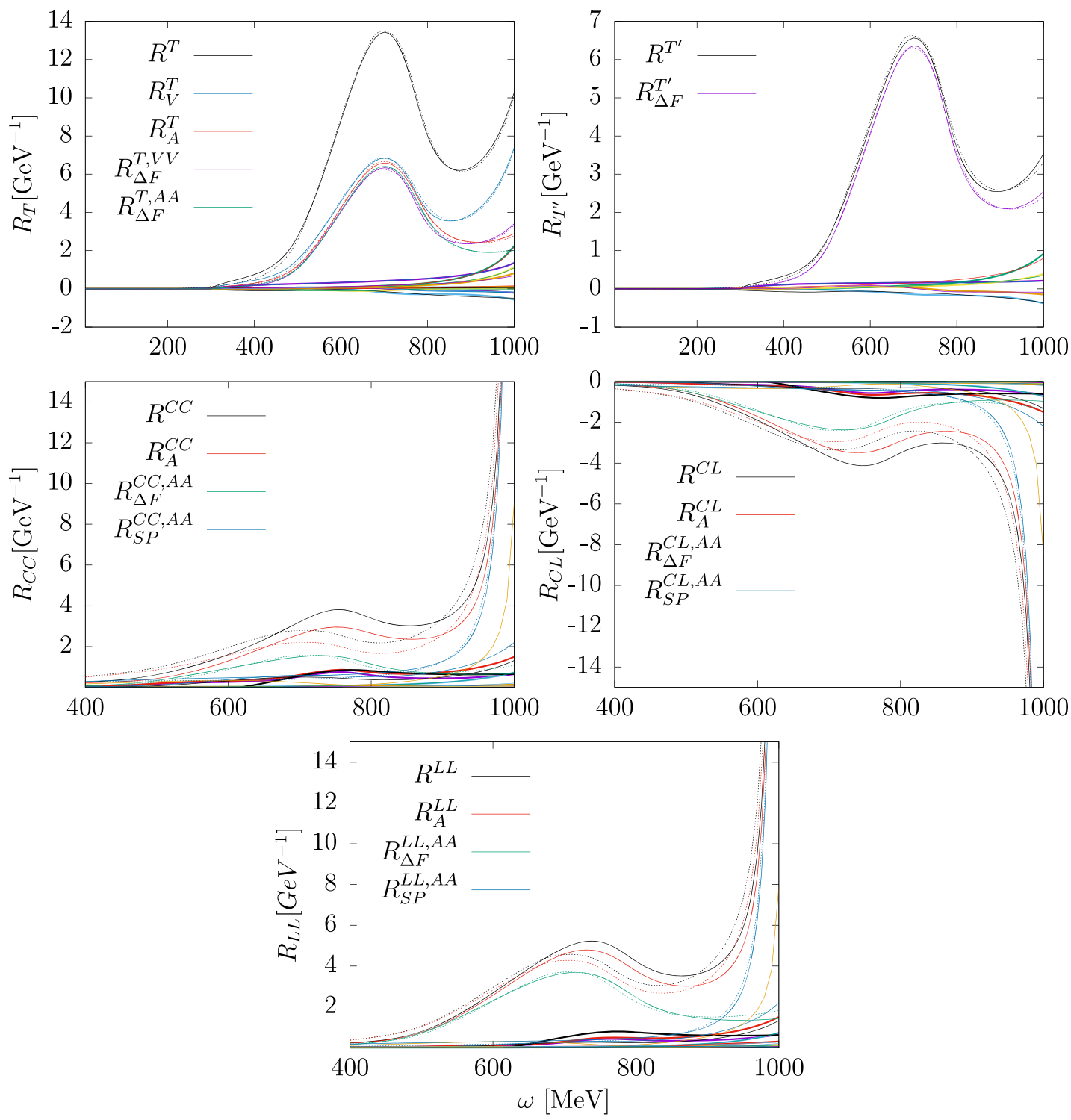

FIG. 4. The same as Fig. 3 for $q=1000 \mathrm{MeV} / \mathrm{c}$. 

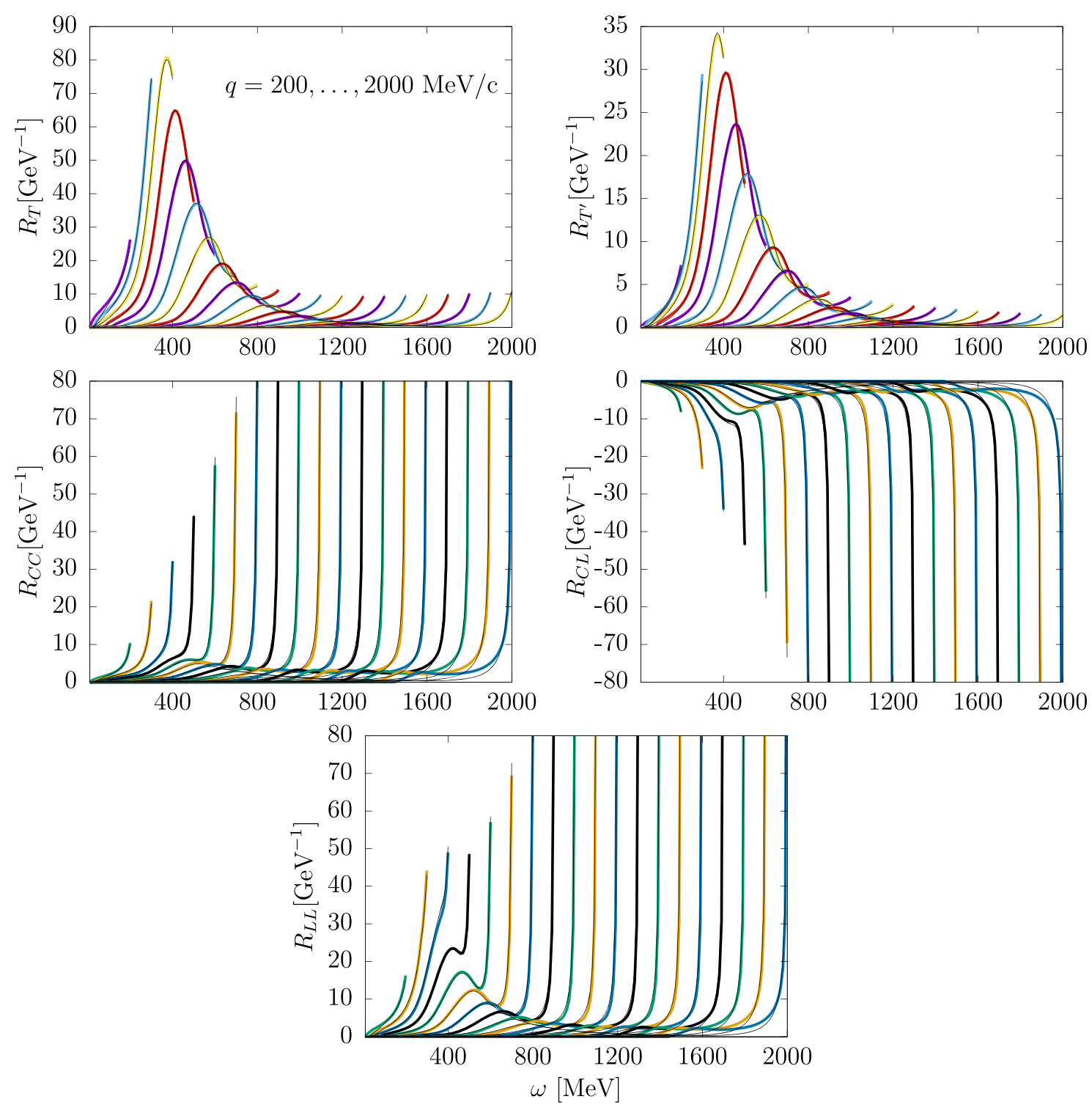

FIG. 5. Comparison of the five $2 \mathrm{p} 2 \mathrm{~h}$ response functions for $\mathrm{CC}$ neutrino scattering from ${ }^{12} \mathrm{C}$, computed with the RMF model and with the semiempirical formula. They are plotted as a function of $\omega$ for fixed values of thew momentum transfer $q=200, \ldots, 2000 \mathrm{MeV} / \mathrm{c}$.

The greatest utility of the SE formula is to calculate the $2 \mathrm{p} 2 \mathrm{~h}$ interaction with neutrinos, since the neutrino flux implies an integration on the incident energy. The integrated cross section in the flux is shown in Fig. 7. There, we show the transverse and longitudinal contributions to the cross section with and without $2 \mathrm{p} 2 \mathrm{~h}$ MEC. The effect of MEC responses, computed with the SE formula, is to increase the cross section of about $\sim 20 \%$, depending on the kinematics. Note that these results have been obtained using $C_{A}^{5}(0)=1.2$ for the axial $\Delta$ coupling. But in Ref. [59], it was found that a value of 0.89 was more adequate according to the pion emission data. If this value is used with the semiempirical formula, the effect of the axial MEC contribution in Fig. 7 would be reduced by almost one-half. Note that the L contribution of the MEC is very small and almost negligible and could be omitted in the calculation.

To visualize the quality of the semiempirical formulas, we show in Fig. 8 the quotient between the semiempirical formula (se) and the exact result (th), for $q=500 \mathrm{MeV} / \mathrm{c}$ as a function of $\omega$. In the zones dominated by the transverse responses $T$ and $T^{\prime}$ at the peak of the delta, the quotient is very close to 1 . For the $\omega$ values where the responses are appreciable, the quotient is practically 1 because the coefficients have been adjusted. The quotient deviates from 1 only for $\omega$ values where the responses are not important nor negligible.

The semiempirical formula also allows studying the relative behavior between the different contributions or subresponses. In particular, one can find relations between 

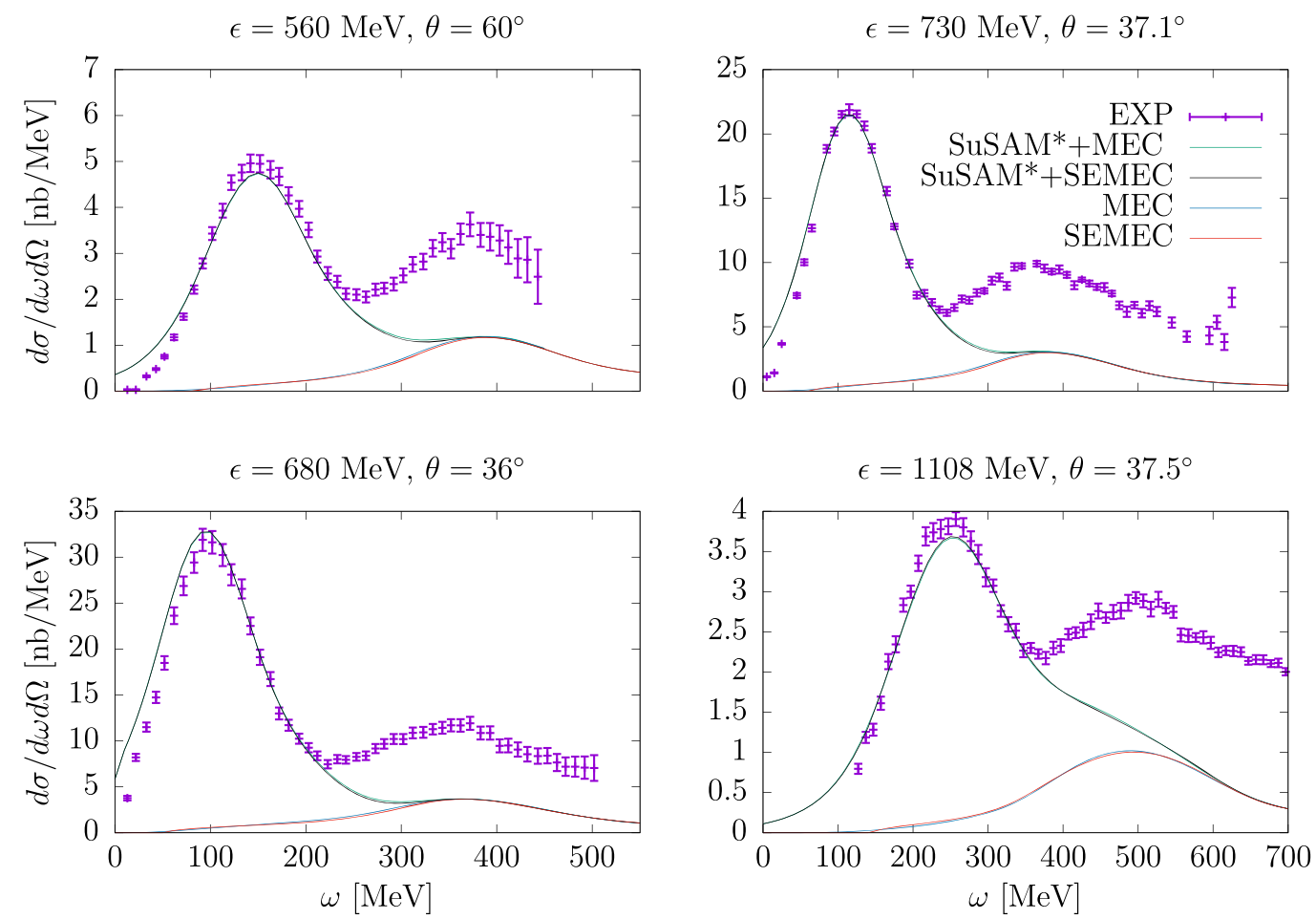

FIG. 6. Cross section for $\left(e, e^{\prime}\right)$ scattering off ${ }^{12} \mathrm{C}$ for various kinematics as a function of omega. The quasielastic contribution with emission of a particle is shown using the SuSAM* model of Ref. [53] and the contribution of channel 2p2h, calculated both with the exact RMF (MEC) and with the semiempirical formula (SE-MEC). Experimental data are from Refs. [69-71].
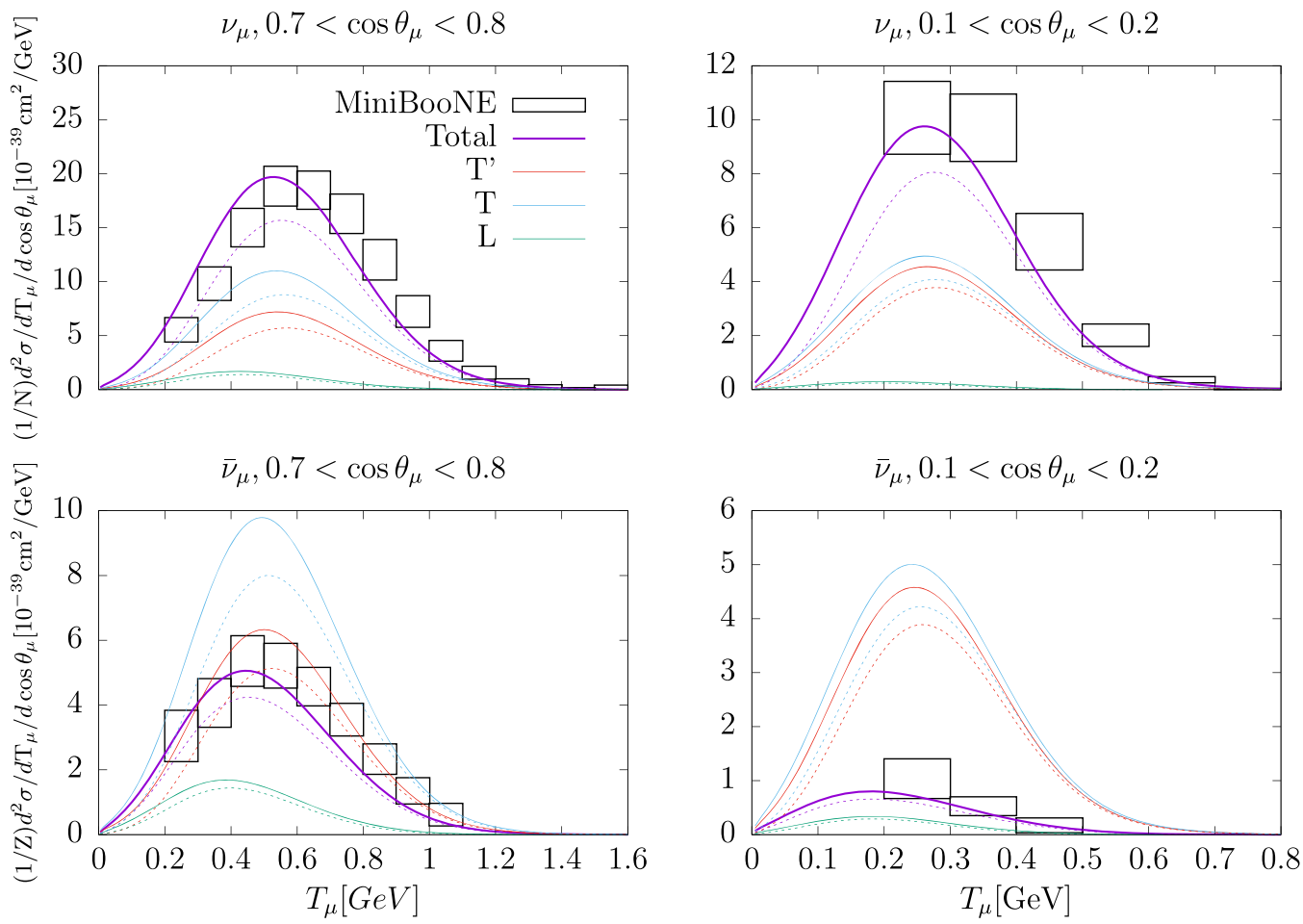

FIG. 7. Quasielastic neutrino and antineutrino, differential cross section integrated over the neutrino flux of the MiniBooNE experiment for selected kinematics of the scattering angle bins. we show the separate $T$, $T^{\prime}$ and longitudinal responses $(L=\mathrm{CC}+\mathrm{CL}+\mathrm{LL})$ with and without MEC. The curves without MEC (dotted lines) have been computed using the SuSAM* model of Ref. [53]. The curves with MEC (solid lines) have been calculated with the SE formula of the 2p2h responses. Experimental data from Refs. [11,12]. 

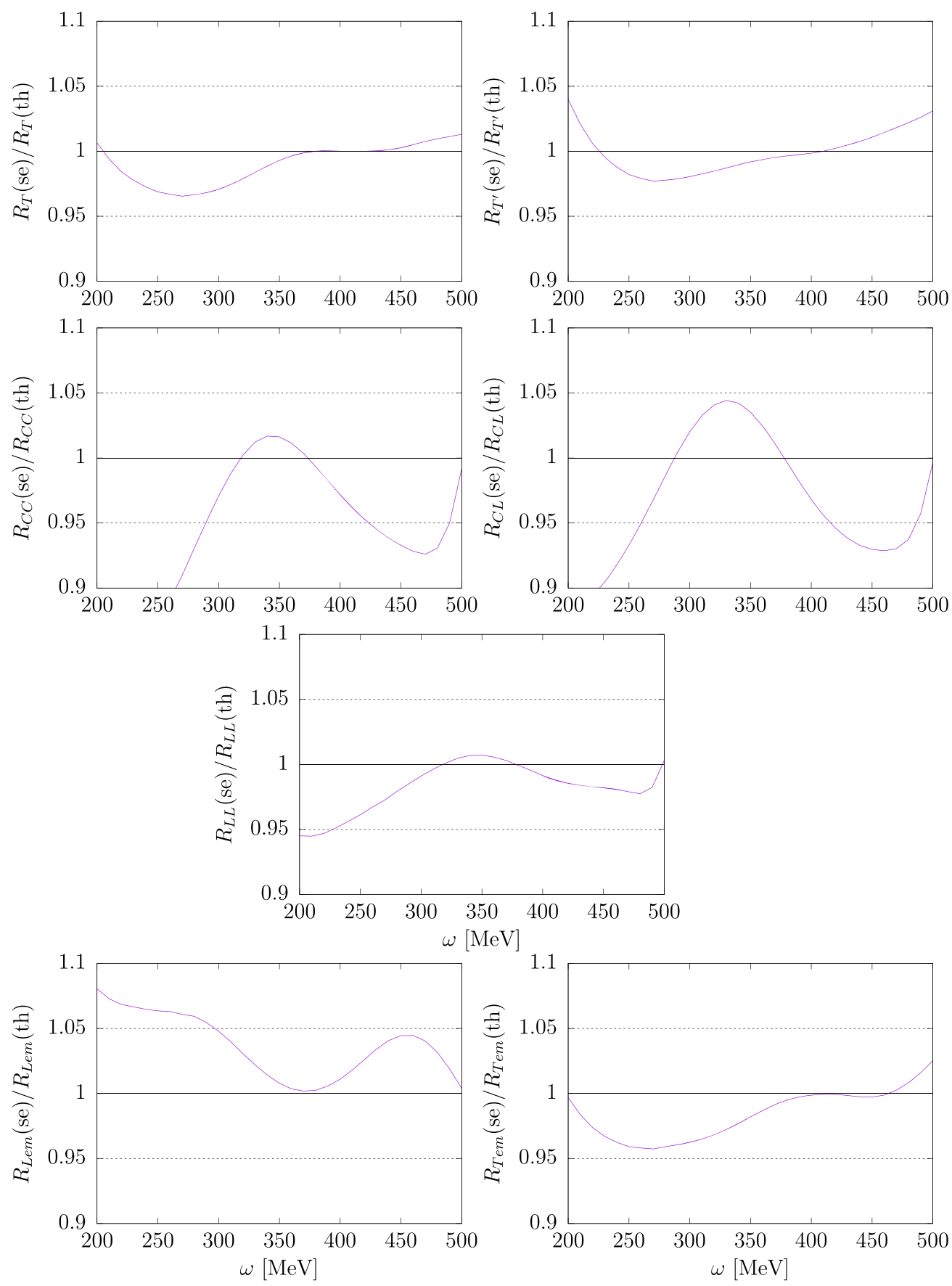

FIG. 8. Quotient between the semi-empirical (se) and theoretical (th) 2p2h response functions for CC neutrino and electron scattering. For $q=500 \mathrm{MeV} / \mathrm{c}$ as a function of $\omega$.

the dominant subresponses $R_{\Delta F}^{T V V}, R_{\Delta F}^{T^{\prime} V A}$, and $R_{\Delta F}^{T A A}$ as follows. First, note that the only difference between the vector and axial parts of the $\Delta F$ current, Eq. (31), is in the electroweak vertex (41), $\Gamma^{\beta \mu}(Q)=\Gamma_{V}^{\beta \mu}(Q)+\Gamma_{A}^{\beta \mu}(Q)$, with

$$
\begin{aligned}
\Gamma_{V}^{\beta \mu}(Q) & =\frac{C_{3}^{V}}{m_{N}}\left(g^{\beta \mu} Q-Q^{\beta} \gamma^{\mu}\right) \gamma_{5}, \\
\Gamma_{A}^{\beta \mu}(Q) & =C_{5}^{A} g^{\beta \mu},
\end{aligned}
$$

Then, the vector current $\Delta F$ is expected to behave roughly like $q / m$ with respect to the axial current. As a consequence, the coefficients of the semiempirical $R_{\Delta F}^{T V V}$ and $R_{\Delta F}^{T^{\prime} V A}$ responses would contain a factor $(q / m)^{2}$ and $q / m$, respectively, with respect to the $R_{\Delta F}^{T A A}$ coefficients. Therefore, if we define the coefficients

$$
\tilde{C}_{1, V i}^{\prime} \equiv \frac{\tilde{C}_{1, V i}}{\left(q / m_{N}\right)^{2}}, \quad \tilde{C}_{1, V A i}^{\prime} \equiv \frac{\tilde{C}_{1, V A i}}{\left(q / m_{N}\right)}
$$



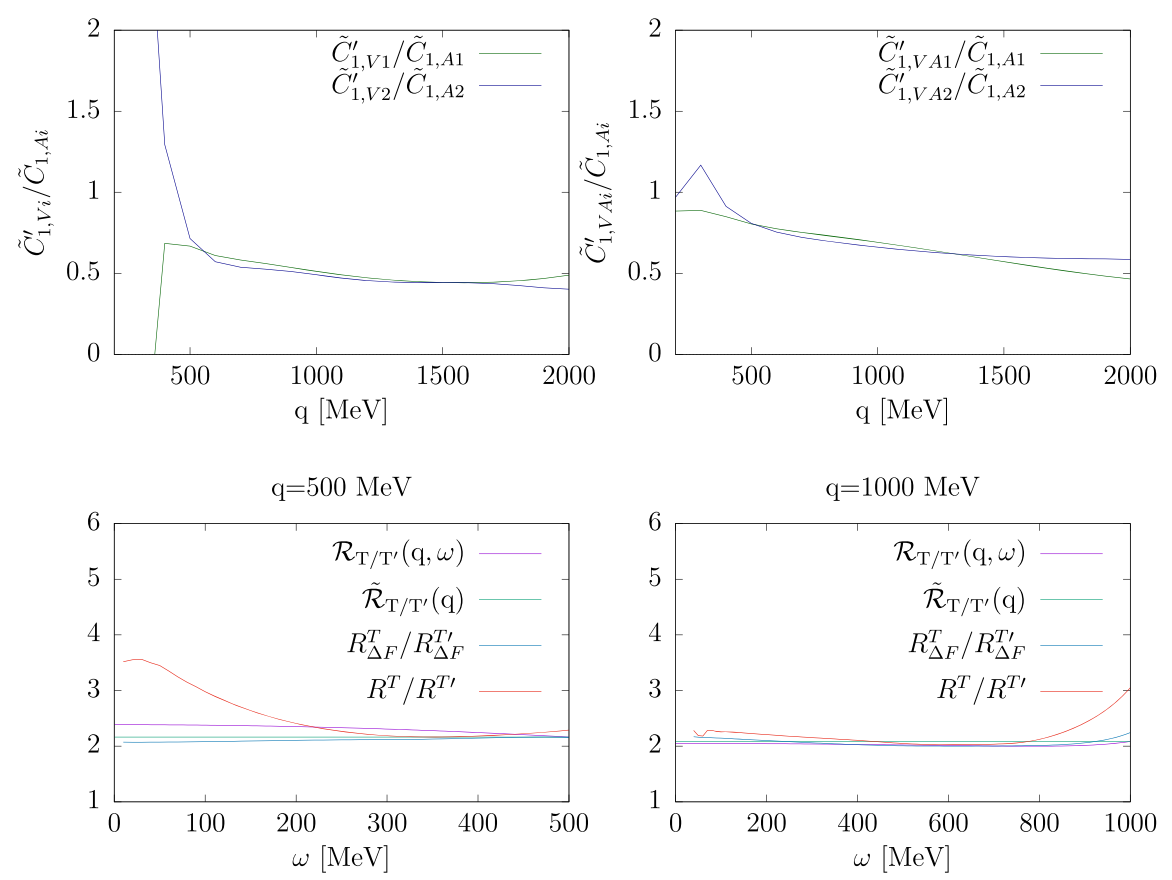

FIG. 9. Top: relation between the coefficients of the $\Delta F$ subresponses $R_{\Delta F}^{T V V}$ and $R_{\Delta F}^{T^{\prime} V A}$, with respect to $R_{\Delta F}^{T A A}$. Bottom: comparison of the quotient $R^{T} / R^{T^{\prime}}$ with several approximations (see the text). The $\mathcal{R}_{T / T^{\prime}}$ and $\tilde{\mathcal{R}}_{T / T^{\prime}}$ functions have been obtained from the semiempirical formulas of the $\Delta F$ subresponses.

then one expects the quotients $\tilde{C}_{1, V i}^{\prime} / \tilde{C}_{1, A i}$ and $\tilde{C}_{1, V A i}^{\prime} / \tilde{C}_{1, A i}$ to be approximately independent of $q$. This is shown in Fig. 9, in which we plot these quotients and see that they are roughly

$$
\begin{gathered}
\frac{\tilde{C}_{1, V i}^{\prime}}{\tilde{C}_{1, A i}} \simeq \frac{1}{2} \\
\frac{\tilde{C}_{1, V A i}^{\prime}}{\tilde{C}_{1, A i}} \simeq \frac{1}{\sqrt{2}} .
\end{gathered}
$$

If we neglect the small contributions of the $S P$ and $\Delta \mathrm{B}$ diagrams, the semiempirical formulas (66), (72), and (78) allow ua to make quantitative estimations of relation between the $T$ and $T^{\prime}$ responses. In fact, the following approximate formulas can be obtained between $R^{T, V V}$ and $R^{T, A A}$,

$$
\frac{R^{T, A A}}{R^{T, V V}} \simeq \frac{R_{\Delta F}^{T, A A}}{R_{\Delta F}^{T, V V}} \simeq \frac{\left(C_{5}^{A}\right)^{2}}{\left(C_{3}^{V}\right)^{2} \frac{1}{2}\left(\frac{q}{m_{N}}\right)^{2}},
$$

as can the following relation between the $T$ and $T^{\prime}$ responses.

$$
\begin{aligned}
\frac{R^{T}}{R^{T^{\prime}}} & \simeq \frac{R_{\Delta F}^{T}}{R_{\Delta F}^{T^{\prime}}} \simeq \frac{\left(C_{3}^{V}\right)^{2} \frac{1}{2}\left(\frac{q}{m_{N}}\right)^{2}+\left(C_{5}^{A}\right)^{2}}{C_{3}^{V} C_{5}^{A} \frac{1}{\sqrt{2}}\left(\frac{q}{m_{N}}\right)} \\
& \equiv \mathcal{R}_{T / T^{\prime}}(q, \omega),
\end{aligned}
$$

where we have defined he function $\mathcal{R}_{T / T^{\prime}}(q, \omega)$ that represents the approximate quotient between the subresponses $T$ and $T^{\prime}$ for the $\Delta F$ diagrams. To obtain Eq. (106), we have used the empirical relations (103) and (104) between the corresponding coefficients, and we have assumed that the averaged $\Delta$ propagators are similar for the $V V, A A$, and $V A$ responses, and they cancel out in the numerator and denominator. The function $\mathcal{R}$ depends on $\omega$ through the $Q^{2}$ dependence of the $\Delta$ form factors $C_{3}^{V}\left(Q^{2}\right)$ and $C_{5}^{A}\left(Q^{2}\right)$. The comparison between this relationship and the exact result is also shown in Fig. 9 for two values of the momentum transfer.

If we also assume that the form factors have an approximately similar dependence on $Q^{2}$, and that this dependence is canceled in the numerator and the denominator, we can simply use the values at the origin, $Q^{2}=0$, of the form factors to obtain a simple approximate formula for the relationship between $\mathrm{T}$ and $\mathrm{T}$ ' responses. In fact, inserting the values $C_{5}^{A}(0)=1.2$, and $C_{3}^{V}(0)=2.13$ in Eq. (106), we can write 


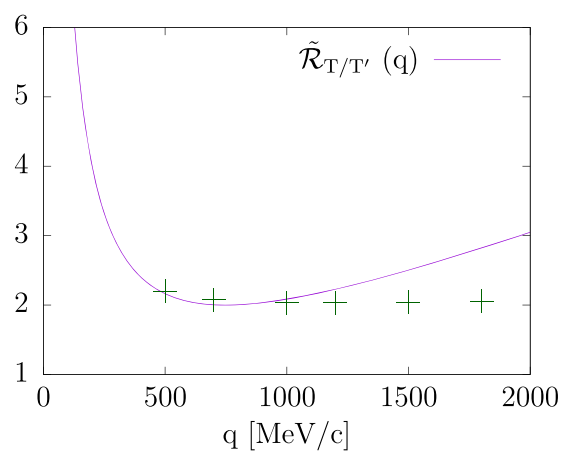

FIG. 10. The semiempirical approximation $\tilde{\mathcal{R}}_{T / T^{\prime}}$ of the quotient between the $T$ and $T^{\prime}$ responses, as a function of $q$.

$$
\frac{R^{T}}{R^{T^{\prime}}} \simeq \frac{(2.13)^{2} \frac{1}{2}\left(\frac{q}{m_{N}}\right)^{2}+(1.2)^{2}}{2.13 \times 1.2 \frac{1}{\sqrt{2}}\left(\frac{q}{m_{N}}\right)} \equiv \tilde{\mathcal{R}}_{T / T^{\prime}}(q)
$$

Note that this approximation does not depend on $\omega$, and this relation is compared in Fig. 10 with the exact result computed for $\omega$ at the maximum of $\Delta$ peak. We see that the formula (107) is valid for $q$ between 500 and $1100 \mathrm{MeV} / \mathrm{c}$, where $\tilde{\mathcal{R}}_{T / T^{\prime}} \simeq 2$. Note that this value depends on the value of $C_{A}^{5}$, and here we have used 1.2.
For larger $q$, the approximations fail because in this region the dominance of the $\Delta F$ starts to decay.

Note that Eqs. (106) and (107) relate $R^{T}$ and $R^{T^{\prime}}$, similarly to Eqs. $(8,9)$ of Ref. [19], but with the $\Delta$ form factors instead of the nucleon form factors, and with similar kinematic factors. In our case, we see, from Figs. 9 and 10, that this relationship is approximately $R^{T} \simeq 2 R^{T^{\prime}}$ in the region of the $\Delta$ peak. This opens the way to determine the $2 \mathrm{p} 2 \mathrm{~h}$ response of neutrinos from fits of the corresponding response in the electromagnetic channel.

Finally, in this work, we have considered the noninteracting free $\Delta$ in the MEC. The semiempirical formula can be extended to include the case of a $\Delta$ interacting with the mean field $[56,72]$. In this case, the $\Delta$ acquires effective mass, $M_{\Delta}^{*}$, and vector energy, $E_{v}^{\Delta}$ (see Appendix A). In the simplest case of universal coupling, the $\Delta$ scalar and vector energies are the same as those of the nucleon. In Fig. 11, we show the effect of including the $\Delta$ self-energy for universal coupling, compared to the case of free $\Delta$. In Fig. 11, we also compare with the extended semiempirical formula of Appendix A. The effect of the $\Delta$ interaction is a $q$-dependent shift, from $-50 \mathrm{MeV}$, for small $q$, up to +50 , for large $q$. The height of the transverse response decreases for low $q$ by $\simeq 30 \%$. The semiempirical formula allows us to easily study the dependence of the responses for other values of the $\Delta$ interaction with the mean field.
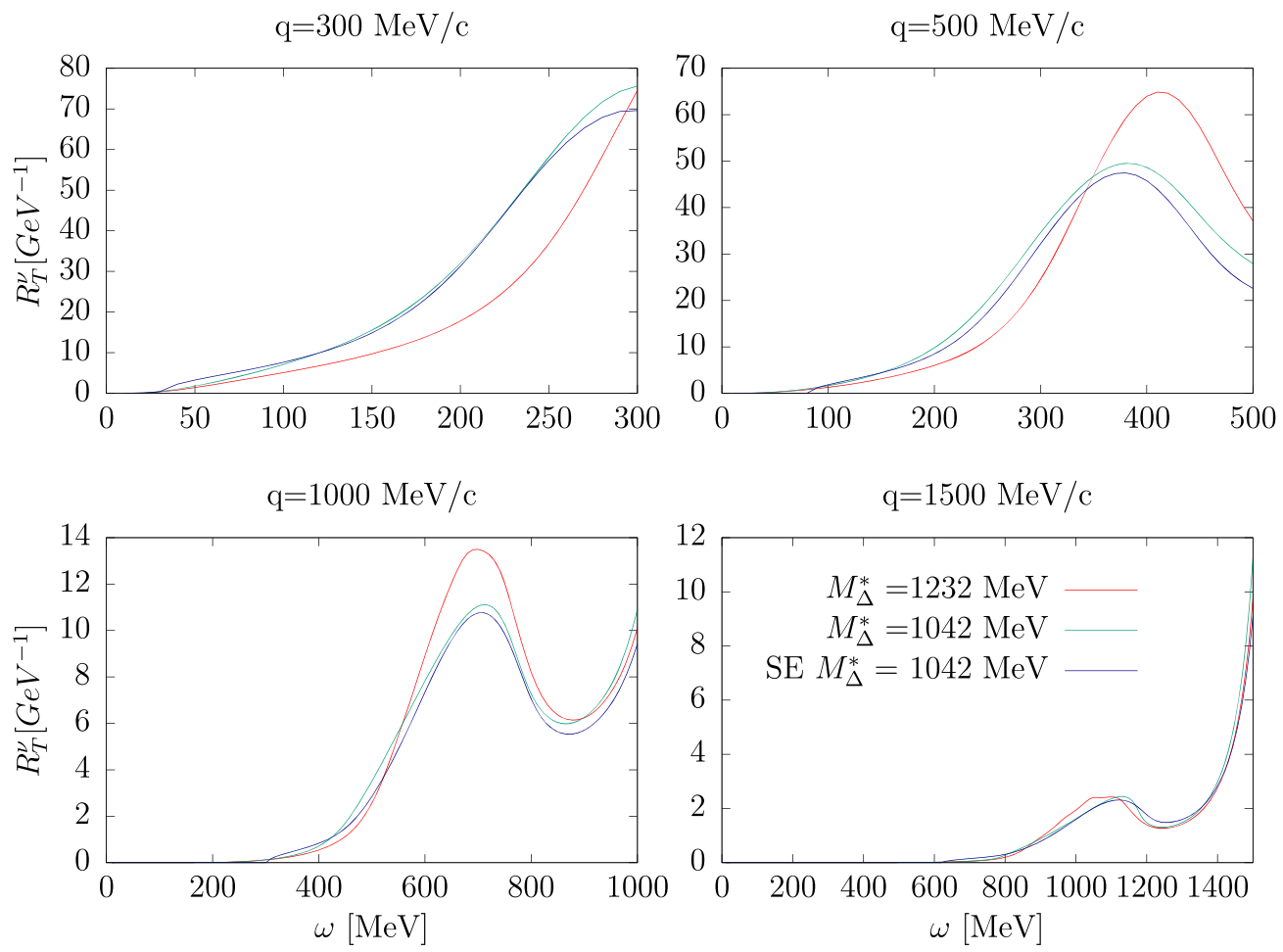

FIG. 11. Comparison of the transverse response functions including or not the $\Delta$ self-energy in the MEC, in the case of universal coupling, $E_{v}^{\Delta}=E_{v}$, and $M_{\Delta}^{*}=1042 \mathrm{MeV}$. The results of the modified semiempirical formula for interacting $\Delta$ are also shown. 


\section{CONCLUSIONS}

In this article, we have proposed a semiempirical formula to approximately calculate the $2 \mathrm{p} 2 \mathrm{~h}$ responses in neutrino scattering. The formula is based on classifying the contribution of Feynman diagrams of MEC with a similar structure, in terms of the same electroweak form factor and the same number of delta-forward propagators, obtaining in our case six contributions, or subresponses, from our three types of diagrams: delta forward, seagull pionic, and delta backward.

It is proposed that each subresponse is the product of the phase-space function of two particles, electroweak form factors, and averaged delta propagators, multiplied by coefficients that only depend on $q$. These coefficients are fitted with a RMF model of nuclear matter for the $2 \mathrm{p} 2 \mathrm{~h}$ responses. In the SE formula, we use an approximation of the phase space (frozen nucleon approximation) and a model for the average propagator of the delta-forward current.

The hypothesis that the coefficients do not depend on $\omega$ is generally fulfilled, with two minor exceptions in the transverse SP subresponses, where a corrective term with a second-degree polynomial in $\omega$ is added. Altogether, we have shown that the dependence on $\omega$ of the $2 \mathrm{p} 2 \mathrm{~h}$ responses comes mainly from these three elements: phase space, form factor, and averaged propagator of the delta forward, and that is it.

Having extracted in the subresponses everything that can be factorized, the semiempirical formulas explicitly contain the dependence on the Fermi momentum, the number of particles, the effective mass and vector energy of the RMF, the electroweak form factors, and the coupling constants, in addition to the explicit dependence on $q$ and $\omega$ through the phase space and the averaged $\Delta$ propagator.

The semiempirical formula has been obtained for free $\Delta$, but then we have generalized it to include a $\Delta$ interacting with the mean field, which already depends on the $\Delta$ effective mass and its vector energy.

An advantage of the semiempirical formula is that it provides the $2 \mathrm{p} 2 \mathrm{~h}$ responses in a model in an easily reproducible way and that it also allows many of the model's parameters to be varied at will.

The semiempirical formula could be extended by including more diagrams or contributions to the MEC, for example, $\rho$-meson exchange, correlation diagrams, and excitation of other nucleon resonances, simply by adding the corresponding form factors, couplings, and propagators to construct the subresponses of the new contributions. The SE formula could also be fitted with other models to recalculate the coefficients.

The SE formulas are promising for application in neutrino calculations and event simulators because they are analytical and allow modifying physical parameters of the model. We are currently studying other nuclei, and we have checked that the formula is valid in symmetric nuclei,
$N=Z$, with the same coefficients of this article, and only the averaged $\Delta$ propagator must be modified by readjusting the effective width and shift.

In the future, we will apply the semiempirical formula to the case of asymmetric matter, $N \neq Z$, which is of interest for current and future neutrino experiments, since in this case the emission channels of pp, pn, and nn have to be treated separately.

\section{ACKNOWLEDGMENTS}

This work has been supported by the Spanish Agencia Estatal de investigacion (DOI 10.13039/501100011033, Grants No. FIS2017-85053-C2-1-P and No. PID2020114767 GB-I00), the Junta de Andalucia (Grant No. FQM-225), and the European Regional Development Funds (Grant No. A-FQM-390-UGR20). V. L. M.-C. acknowledges a contract funded by Agencia Estatal de Investigacion and European Social Fund.

\section{APPENDIX A: SEMIEMPIRICAL FORMULA FOR $\triangle$ IN THE MEDIUM}

In this Appendix, we explain how the semiempirical formula must be modified to include the effective mass of the $\Delta$ and its vector energy. In this work, we have neglected the interaction of the $\Delta$, treating it as a free particle. If we assume that the $\Delta$ interacts with the scalar and vector fields of the RMF, the $\Delta$ acquires an effective mass and a vector energy $[56,72]$,

$$
\begin{gathered}
M_{\Delta}^{*}=M_{\Delta}-g_{s}^{\Delta} \phi_{0} \\
E_{\mathrm{RMF}}^{\Delta}=E^{\Delta}+E_{v}^{\Delta}
\end{gathered}
$$

with $E^{\Delta}=\sqrt{p_{\Delta}^{2}+M_{\Delta}^{* 2}}$ is the on-shell energy of the intermediate $\Delta$ isobar with momentum $\mathbf{p}_{\Delta}$.

In this case, the delta current would be modified by substituting the propagator of the free delta, Eq. (45), for the propagator in the medium, which is the following [56]:

$$
G_{\alpha \beta}(P)=\frac{\mathcal{P}_{\alpha \beta}\left(P^{*}\right)}{P^{* 2}-M_{\Delta}^{* 2}+i M_{\Delta}^{*} \Gamma_{\Delta}\left(P^{* 2}\right)+\frac{\Gamma_{\Delta}\left(P^{* 2}\right)^{2}}{4}} .
$$

The projector $\mathcal{P}_{\alpha \beta}\left(P^{*}\right)$ is now

$$
\begin{aligned}
\mathcal{P}_{\alpha \beta}\left(P^{*}\right)= & -\left(\boldsymbol{P}^{*}+M_{\Delta}^{*}\right) \\
& \times\left[g_{\alpha \beta}-\frac{1}{3} \gamma_{\alpha} \gamma_{\beta}-\frac{2}{3} \frac{P_{\alpha}^{*} P_{\beta}^{*}}{M_{\Delta}^{* 2}}+\frac{1}{3} \frac{P_{\alpha}^{*} \gamma_{\beta}-P_{\beta}^{*} \gamma_{\alpha}}{M_{\Delta}^{*}}\right],
\end{aligned}
$$

where $P^{* \mu}=P^{\mu}-\delta_{\mu, 0} E_{v}^{\Delta}$. With the $\Delta$ in the medium, the peak position of the delta-forward response is expected at

$$
\omega=\sqrt{q^{2}+M_{\Delta}^{* 2}}-m_{N}^{*}-E_{v}+E_{v}^{\Delta} .
$$


In the particular case of a free $\Delta$, we recover the original position of the peak at

$$
\omega=\sqrt{q^{2}+M_{\Delta}^{2}}-m_{N}^{*}-E_{v}
$$

We see that the position of the $\Delta$-forward peak depends on $q$ and the values of the vector energies and the effective masses. In this case, the averaged $\Delta$ propagator, Eq. (54), is still calculated with the same formula, but changing the values of the parameters $a$ and $b$ to include the vector energy and the effective mass of the $\Delta$ in the mean field

$$
\begin{gathered}
a \equiv m_{N}^{* 2}+\left(\omega+E_{v}-E_{v}^{\Delta}\right)^{2}-q^{2} \\
+2 m_{N}^{*}\left(\omega+E_{v}-E_{v}^{\Delta}+\Sigma\right)-M_{\Delta}^{* 2}+\frac{\Gamma^{2}}{4} \\
b \equiv M_{\Delta}^{*} \Gamma .
\end{gathered}
$$

Note that the $\Delta$ effective mass also appears in the numerator of the $\Delta$ propagator, and this modifies the values of the matrix elements of the delta-forward current operator and the values of the responses. We have computed the new responses. For simplicity, we have assumed universal coupling, where the scalar and vector self-energies of the $\Delta$ are the same as the nucleon, and also the case where only the vector energies are equal. For universal coupling, $E_{v}^{\Delta}=E_{v}=141 \mathrm{MeV}, \quad$ and $M_{\Delta}^{*}=1042 \mathrm{MeV}$. We have found that the semiempirical formula can be easily modified to include these cases in the following lines, by replacing the parameters of the $\Delta$ forward responses

$$
\begin{gathered}
\tilde{C}_{1, i j} \rightarrow\left(\frac{M_{\Delta}^{*}}{M_{\Delta}}\right)^{2} \tilde{C}_{1, i j} \\
\tilde{C}_{4, i j} \rightarrow\left(\frac{M_{\Delta}^{*}}{M_{\Delta}}\right) \tilde{C}_{4, i j} \\
\tilde{C}_{5, i j} \rightarrow\left(\frac{M_{\Delta}^{*}}{M_{\Delta}}\right) \tilde{C}_{5, i j},
\end{gathered}
$$

where $i=V, A, V A$, and $j=1,2$. The rest of the parameters of the SE formula are not modified.

\section{APPENDIX B: TABLES OF COEFFICIENTS OF THE SEMIEMPIRICAL MEC FORMULAS}

In this Appendix, we provide the values of the coefficients, $\tilde{C}_{i}(q)$, of the semiempirical MEC responses for $q=200 \mathrm{MeV} / \mathrm{c}$ up to $q=2000 \mathrm{MeC} / \mathrm{c}$ in steps of $\Delta q=100 \mathrm{MeV} / \mathrm{c}$. These tables can be interpolated to compute them for other $q$ values. Alternatively, polynomial parametrizations of these coefficient are provided in Appendix C. The tables are included as additional material in plain text [73].

In Table I, we give the averaged width, $\Gamma(q)$, and averaged shift, $\Sigma(q)$, of the averaged $\Delta$ propagator, $G_{\text {av }}(q, \omega)$, for each one of the response functions of the kind $V V, A A$, or $V A$.

The coefficients of the SE MEC are provided in Tables II (for the $R_{T}^{V V}$ response function), III (for $R_{T}^{A A}$ ), IV (for $R_{C C}^{V V}$ ), $\mathrm{V}$ (for $R_{C C}^{A A}$ ), VI (for $R_{C L}^{A A}$ ), VII (for $R_{L L}^{A A}$ ), and VIII (for $R_{T^{\prime}}^{V A}$ ).

TABLE I. Values of the $\Sigma$ and $\Gamma$ parameters of the averaged $\Delta$ propagator in the vector, axial, and vector-axial responses.

\begin{tabular}{lcccccc}
\hline \hline $\mathrm{q}$ & $\Sigma_{V}$ & $\Gamma_{V}$ & $\Sigma_{A}$ & $\Gamma_{A}$ & $\Sigma_{V A}$ & $\Gamma_{V A}$ \\
\hline 200 & 429.56 & 411.88 & 370.98 & 354.59 & -414.99 & 392.89 \\
300 & 78.986 & 185.09 & 56.717 & 213.63 & 68.141 & 202.39 \\
400 & 91.125 & 166.59 & 77.657 & 105.54 & 86.064 & 114.85 \\
500 & 98.153 & 110.12 & 79.080 & 108.77 & 89.183 & 109.51 \\
600 & 102.60 & 101.32 & 81.094 & 112.29 & 92.511 & 104.93 \\
700 & 107.11 & 95.220 & 83.883 & 112.49 & 96.193 & 100.76 \\
800 & 11.70 & 89.771 & 87.198 & 111.84 & 100.14 & 96.588 \\
900 & 116.35 & 84.322 & 90.914 & 11.16 & 104.23 & 92.175 \\
1000 & 120.93 & 79.042 & 94.968 & 110.81 & 108.29 & 87.599 \\
1100 & 125.48 & 74.275 & 99.373 & 110.79 & 112.16 & 82.932 \\
1200 & 130.43 & 70.570 & 104.18 & 110.83 & 115.85 & 78.412 \\
1300 & 136.28 & 67.973 & 109.36 & 110.74 & 119.34 & 74.053 \\
1400 & 143.48 & 66.060 & 114.90 & 110.19 & 122.58 & 69.760 \\
1500 & 152.32 & 64.801 & 120.78 & 108.83 & 125.39 & 65.752 \\
1600 & 163.36 & 63.297 & 126.84 & 106.84 & 127.73 & 61.963 \\
1700 & 177.02 & 60.783 & 132.90 & 103.96 & 129.37 & 58.403 \\
1800 & 193.85 & 56.980 & 138.53 & 100.53 & 130.07 & 55.603 \\
1900 & 214.45 & 51.489 & 143.10 & 97.028 & 129.72 & 53.580 \\
2000 & 239.30 & 44.367 & 145.85 & 94.244 & 128.13 & 52.692 \\
\hline \hline
\end{tabular}


TABLE II. Coefficients $\tilde{C}_{i}(q)$ of the $R_{T}^{V V}$ response function in the semiempirical MEC formulas.

\begin{tabular}{|c|c|c|c|c|c|c|c|c|c|}
\hline$q$ & $\tilde{C}_{1, V 1}$ & $\tilde{C}_{1, V 2}$ & $\tilde{C}_{2, V}$ & $\tilde{C}_{3, V}$ & $\tilde{C}_{4, V 1}$ & $\tilde{C}_{4, V 2}$ & $\tilde{C}_{5, V 1}$ & $\tilde{C}_{5, V 2}$ & $\tilde{C}_{6, V}$ \\
\hline 200 & -5.6455 & 4.7254 & 324.95 & 0.8821 & 19.976 & -11.856 & -0.0750 & -0.1005 & 4.9442 \\
\hline 300 & -2.3128 & 7.0572 & 277.05 & 1.6642 & 6.7713 & -8.4869 & -0.2942 & -0.1056 & 8.2559 \\
\hline 400 & 2.8522 & 5.6335 & 237.33 & 2.3559 & -3.6706 & -2.9271 & -0.4448 & -0.1328 & 10.339 \\
\hline 500 & 4.6067 & 5.6932 & 207.99 & 2.9384 & -4.6034 & -2.4378 & -0.4102 & -0.2456 & 11.498 \\
\hline 600 & 6.3810 & 7.3485 & 187.15 & 3.4589 & -5.3142 & -2.0497 & -0.4561 & -0.3564 & 12.148 \\
\hline 700 & 8.6406 & 10.267 & 172.73 & 3.9693 & -6.3515 & -1.3441 & -0.5438 & -0.4778 & 12.560 \\
\hline 800 & 11.229 & 14.080 & 163.24 & 4.5053 & -7.4147 & -0.2435 & -0.6380 & -0.5912 & 12.867 \\
\hline 900 & 14.017 & 18.385 & 157.49 & 5.0833 & -8.3548 & 1.2379 & -0.7307 & -0.6812 & 13.126 \\
\hline 1000 & 16.967 & 22.966 & 154.81 & 5.7084 & -9.1218 & 3.0420 & -0.8307 & -0.7465 & 13.364 \\
\hline 1100 & 20.125 & 27.880 & 154.72 & 6.3784 & -9.6915 & 5.1327 & -0.9510 & -0.7926 & 13.588 \\
\hline 1200 & 23.578 & 33.383 & 156.96 & 7.0876 & -10.030 & 7.5290 & -1.1024 & -0.8230 & 13.803 \\
\hline 1300 & 27.448 & 39.763 & 161.41 & 7.8296 & -10.104 & 10.278 & -1.2937 & -0.8428 & 14.007 \\
\hline 1400 & 31.840 & 47.170 & 168.06 & 8.5967 & -9.8987 & 13.412 & -1.5289 & -0.8607 & 14.200 \\
\hline 1500 & 36.836 & 55.518 & 177.07 & 9.3820 & -9.4335 & 16.925 & -1.8104 & -0.8945 & 14.380 \\
\hline 1600 & 42.528 & 64.336 & 188.67 & 10.179 & -8.7568 & 20.743 & -2.1399 & -0.9698 & 14.545 \\
\hline 1700 & 49.031 & 72.952 & 203.28 & 10.980 & -7.9275 & 24.742 & -2.5173 & -1.1085 & 14.696 \\
\hline 1800 & 56.482 & 80.929 & 221.50 & 11.781 & -6.9963 & 28.795 & -2.9423 & -1.3179 & 14.831 \\
\hline 1900 & 65.053 & 88.622 & 244.16 & 12.577 & -5.9638 & 32.999 & -3.4146 & -1.5896 & 14.948 \\
\hline 2000 & 74.960 & 98.238 & 272.46 & 13.365 & -4.7513 & 38.182 & -3.9421 & -2.0225 & 15.050 \\
\hline
\end{tabular}

TABLE III. Coefficients $\tilde{C}_{i}(q)$ of the $R_{T}^{A A}$ response function in the semiempirical MEC formulas.

\begin{tabular}{|c|c|c|c|c|c|c|c|c|c|}
\hline$q$ & $\tilde{C}_{1, A 1}$ & $\tilde{C}_{1, A 2}$ & $\tilde{C}_{2, A}$ & $\tilde{C}_{3, A}$ & $\tilde{C}_{4, A 1}$ & $\tilde{C}_{4, A 2}$ & $\tilde{C}_{5, A 1}$ & $\tilde{C}_{5, A 2}$ & $\tilde{C}_{6, A}$ \\
\hline 200 & 16.329 & 34.206 & 19.944 & 20.615 & 4.1488 & -2.5516 & -2.7292 & 2.6273 & 17.649 \\
\hline 300 & 21.430 & 18.820 & 18.724 & 18.470 & 3.7251 & -0.2755 & -2.3497 & -0.0523 & 16.196 \\
\hline 400 & 22.912 & 23.973 & 16.754 & 15.620 & 2.7107 & 1.3642 & -2.1103 & -1.1070 & 13.495 \\
\hline 500 & 24.292 & 28.051 & 14.811 & 13.089 & 2.4231 & 1.8619 & -2.2025 & -1.5055 & 10.924 \\
\hline 600 & 25.597 & 31.450 & 13.062 & 11.054 & 2.2471 & 2.2170 & -2.3253 & -1.7242 & 8.8130 \\
\hline 700 & 26.669 & 34.336 & 11.520 & 9.4730 & 2.0649 & 2.5229 & -2.3886 & -1.8179 & 7.1491 \\
\hline 800 & 27.590 & 36.844 & 10.172 & 8.2615 & 1.8603 & 2.7740 & -2.3935 & -1.8096 & 5.8390 \\
\hline 900 & 28.405 & 39.083 & 8.9866 & 7.3289 & 1.6293 & 2.9601 & -2.3566 & -1.7396 & 4.7773 \\
\hline 1000 & 29.015 & 41.136 & 7.9396 & 6.6055 & 1.3711 & 3.0905 & -2.2927 & -1.6460 & 3.8973 \\
\hline 1100 & 29.845 & 43.044 & 7.0164 & 6.0317 & 1.0980 & 3.1824 & -2.2107 & -1.5499 & 3.1401 \\
\hline 1200 & 30.530 & 44.799 & 6.1995 & 5.5604 & 0.8124 & 3.2402 & -2.1127 & -1.4694 & 2.4634 \\
\hline 1300 & 31.210 & 46.400 & 5.4813 & 5.1521 & 0.5207 & 3.2635 & -1.9980 & -1.4097 & 1.8359 \\
\hline 1400 & 31.879 & 47.821 & 4.8477 & 4.7766 & 0.2225 & 3.2622 & -1.8677 & -1.3610 & 1.2352 \\
\hline 1500 & 32.511 & 49.056 & 4.2924 & 4.4065 & -0.0743 & 3.2381 & -1.7222 & -1.3142 & 0.6426 \\
\hline 1600 & 33.070 & 50.100 & 3.8069 & 4.0198 & -0.3670 & 3.1950 & -1.5655 & -1.2580 & 0.0461 \\
\hline 1700 & 33.514 & 50.979 & 3.3820 & 3.5983 & -0.6496 & 3.1351 & -1.4033 & -1.1864 & -0.5625 \\
\hline 1800 & 33.803 & 51.770 & 3.0103 & 3.1267 & -0.9176 & 3.0642 & -1.2428 & -1.1018 & -1.1922 \\
\hline 1900 & 33.905 & 52.613 & 2.6875 & 2.5931 & -1.1712 & 2.9866 & -1.0965 & -0.9982 & -1.8438 \\
\hline 2000 & 33.778 & 53.762 & 2.4071 & 1.9874 & -1.3992 & 2.9084 & -0.9692 & -0.8808 & -2.5212 \\
\hline
\end{tabular}


TABLE IV. Coefficients $\tilde{C}_{i}(q)$ of the $R_{C C}^{V V}$ response function in the semiempirical MEC formulas.

\begin{tabular}{|c|c|c|c|c|c|c|c|c|c|}
\hline$q$ & $\tilde{C}_{1, V 1}$ & $\tilde{C}_{1, V 2}$ & $\tilde{C}_{2, V}$ & $\tilde{C}_{3, V}$ & $\tilde{C}_{4, V 1}$ & $\tilde{C}_{4, V 2}$ & $\tilde{C}_{5, V 1}$ & $\tilde{C}_{5, V 2}$ & $\tilde{C}_{6, V}$ \\
\hline 200 & -0.1024 & 0.0854 & 8.5525 & 0.0476 & 0.3211 & -0.1617 & 0.0030 & -0.0135 & 0.0357 \\
\hline 300 & -0.0486 & 0.1498 & 6.7440 & 0.1319 & 0.1593 & -0.1606 & -0.0159 & -0.0211 & 0.1003 \\
\hline 400 & 0.0678 & 0.1505 & 5.4143 & 0.2642 & -0.0385 & -0.0810 & -0.0463 & -0.0248 & 0.1893 \\
\hline 500 & 0.1578 & 0.1882 & 4.5372 & 0.4452 & -0.0496 & -0.0868 & -0.0653 & -0.0422 & 0.3005 \\
\hline 600 & 0.3027 & 0.2904 & 3.9966 & 0.6710 & 0.0294 & -0.0948 & -0.1020 & -0.0694 & 0.4303 \\
\hline 700 & 0.5658 & 0.4717 & 3.6898 & 0.9354 & 0.2210 & -0.0787 & -0.1666 & -0.1106 & 0.5732 \\
\hline 800 & 1.0273 & 0.7286 & 3.5402 & 1.2320 & 0.5934 & -0.0121 & -0.2661 & -0.1706 & 0.7238 \\
\hline 900 & 1.8174 & 1.0250 & 3.4920 & 1.5559 & 1.2182 & 0.1411 & -0.4121 & -0.2582 & 0.8770 \\
\hline 1000 & 3.1690 & 1.2899 & 3.5088 & 1.9032 & 2.1686 & 0.4230 & -0.6208 & -0.3881 & 1.0291 \\
\hline 1100 & 5.5066 & 1.4027 & 3.5654 & 2.2711 & 3.5213 & 0.8816 & -0.9112 & -0.5782 & 1.1773 \\
\hline 1200 & 9.5630 & 1.1584 & 3.6453 & 2.6577 & 5.3523 & 1.5678 & -1.3018 & -0.8488 & 1.3193 \\
\hline 1300 & 16.468 & 0.2226 & 3.7376 & 3.0612 & 7.7257 & 2.5367 & -1.8108 & -1.2243 & 1.4536 \\
\hline 1400 & 27.710 & -1.8853 & 3.8351 & 3.4808 & 10.692 & 3.8489 & -2.4530 & -1.7339 & 1.5790 \\
\hline 1500 & 44.980 & -5.7623 & 3.9328 & 3.9156 & 14.285 & 5.5617 & -3.2416 & -2.4152 & 1.6947 \\
\hline 1600 & 70.073 & -12.111 & 4.0275 & 4.3654 & 18.052 & 7.7114 & -4.1882 & -3.3012 & 1.8002 \\
\hline 1700 & 104.98 & -21.738 & 4.1169 & 4.8302 & 23.426 & 10.286 & -5.3006 & -4.4046 & 1.8959 \\
\hline 1800 & 152.13 & -35.588 & 4.1997 & 5.3101 & 29.007 & 13.208 & -6.5868 & -5.7040 & 1.9812 \\
\hline 1900 & 214.67 & -54.766 & 4.2747 & 5.8056 & 35.314 & 16.426 & -8.0633 & -7.1889 & 2.0576 \\
\hline 2000 & 296.65 & -80.395 & 4.3415 & 6.3170 & 42.457 & 20.217 & -9.7724 & -9.1248 & 2.1249 \\
\hline
\end{tabular}

TABLE V. Coefficients $\tilde{C}_{i}(q)$ of the $R_{C C}^{A A}$ response function in the semiempirical MEC formulas.

\begin{tabular}{lccccccrrrr}
\hline \hline $\mathrm{q}$ & $\tilde{C}_{1, A 1}$ & $\tilde{C}_{1, A 2}$ & $\tilde{C}_{2, A 1}$ & $\tilde{C}_{2, A 2}$ & $\tilde{C}_{3, A}$ & $\tilde{C}_{4, A 1}$ & \multicolumn{1}{c}{$\tilde{C}_{4, A 2}$} & $\tilde{C}_{5, A 1}$ & \multicolumn{1}{c}{$\tilde{C}_{5, A 2}$} & $\tilde{C}_{6, A}$ \\
\hline 200 & 0.4310 & 0.6758 & 238.27 & 9.3999 & 0.3545 & 1.0876 & -50.597 & 0.0504 & -0.0292 & 0.3475 \\
300 & 0.7425 & 0.6100 & 215.77 & 8.4094 & 0.3314 & -8.2958 & -11.926 & 0.0265 & 0.0090 & 0.0575 \\
400 & 1.1073 & 1.3165 & 175.09 & 7.7175 & 0.3006 & -7.1932 & -8.0118 & 0.0064 & 0.0216 & -0.1498 \\
500 & 2.1427 & 2.1218 & 138.76 & 6.9936 & 0.2787 & -4.9007 & -7.7936 & 0.0210 & 0.0146 & -0.3151 \\
600 & 3.1362 & 3.1395 & 109.68 & 6.2763 & 0.2646 & -0.4025 & -8.1195 & 0.0515 & 0.0109 & -0.4585 \\
700 & 4.3281 & 4.3521 & 87.033 & 5.6002 & 0.2544 & 4.6146 & -8.0345 & 0.0965 & 0.0144 & -0.5908 \\
800 & 5.7860 & 5.7550 & 69.523 & 4.9837 & 0.2442 & 10.156 & -7.5209 & 0.1605 & 0.0296 & -0.7057 \\
900 & 7.6019 & 7.3393 & 55.929 & 4.4317 & 0.2305 & 16.577 & -6.5162 & 0.2447 & 0.0588 & -0.8128 \\
1000 & 9.9183 & 9.1025 & 45.328 & 3.9429 & 0.2106 & 24.215 & -4.9410 & 0.3495 & 0.1041 & -0.9201 \\
1100 & 12.958 & 11.016 & 37.025 & 3.5122 & 0.1822 & 33.432 & -2.6731 & 0.4694 & 0.1641 & -1.0171 \\
1200 & 17.041 & 13.007 & 30.456 & 3.1346 & 0.1430 & 44.503 & 0.4040 & 0.5954 & 0.2368 & -1.1184 \\
1300 & 22.616 & 14.950 & 25.230 & 2.8037 & 0.0918 & 57.725 & 4.4288 & 0.7159 & 0.3149 & -1.2640 \\
1400 & 30.266 & 16.646 & 21.041 & 2.5137 & 0.0271 & 73.368 & 9.5238 & 0.8128 & 0.3868 & -1.3986 \\
1500 & 40.702 & 17.843 & 17.664 & 2.2593 & -0.0514 & 91.621 & 15.780 & 0.8644 & 0.4368 & -1.5547 \\
1600 & 54.774 & 18.212 & 14.919 & 2.0359 & -0.1444 & 112.59 & 23.235 & 0.8495 & 0.4422 & -1.7426 \\
1700 & 73.477 & 17.381 & 12.677 & 1.8392 & -0.2522 & 136.52 & 31.950 & 0.7379 & 0.3723 & -1.9275 \\
1800 & 97.967 & 14.957 & 10.833 & 1.6658 & -0.3739 & 163.50 & 41.932 & 0.5062 & 0.1975 & -2.1423 \\
1900 & 129.63 & 10.517 & 9.3095 & 1.5124 & -0.5101 & 193.73 & 53.264 & 0.1143 & -0.1323 & -2.3821 \\
2000 & 170.12 & 3.6516 & 8.0440 & 1.3765 & -0.6595 & 227.56 & 66.185 & -0.4731 & -0.6773 & -2.6522 \\
\hline \hline
\end{tabular}


TABLE VI. Coefficients $\tilde{C}_{i}(q)$ of the $R_{C L}^{A A}$ response function in the semiempirical MEC formulas.

\begin{tabular}{lrrrrrrrrrr}
\hline \hline $\mathrm{q}$ & $\tilde{C}_{1, A 1}$ & $\tilde{C}_{1, A 2}$ & $\tilde{C}_{2, A 1}$ & $\tilde{C}_{2, A 2}$ & $\tilde{C}_{3, A}$ & $\tilde{C}_{4, A 1}$ & $\tilde{C}_{4, A 2}$ & $\tilde{C}_{5, A 1}$ & $\tilde{C}_{5, A 2}$ & $\tilde{C}_{6, A}$ \\
\hline 200 & -1.1765 & -2.5379 & -72.678 & -7.2538 & 0.0806 & 5.5173 & 34.373 & 0.0563 & 0.1300 & -0.7486 \\
300 & -2.1851 & -2.0364 & -91.567 & -7.3504 & -0.1306 & 9.9664 & 13.202 & 0.1902 & -0.0182 & -0.6646 \\
400 & -3.0251 & -3.6397 & -86.641 & -7.0749 & -0.2579 & 8.1204 & 10.129 & 0.1989 & 0.0688 & -0.4324 \\
500 & -4.9236 & -5.0983 & -75.322 & -6.5790 & -0.3193 & 6.9727 & 9.2048 & 0.1870 & 0.1269 & -0.2418 \\
600 & -6.3387 & -6.6909 & -63.463 & -5.9990 & -0.3393 & 2.5570 & 9.3724 & 0.1727 & 0.1655 & -0.1346 \\
700 & -7.8449 & -8.3950 & -52.859 & -5.4096 & -0.3340 & -2.7198 & 9.1389 & 0.1527 & 0.1909 & -0.1047 \\
800 & -9.5543 & -10.225 & -43.896 & -4.8494 & -0.3134 & -8.5236 & 8.4471 & 0.1251 & 0.2045 & -0.1183 \\
900 & -11.573 & -12.190 & -36.470 & -4.3350 & -0.2830 & -15.121 & 7.2693 & 0.0907 & 0.2064 & -0.1513 \\
1000 & -14.046 & -14.312 & -30.392 & -3.8718 & -0.2457 & -22.835 & 5.5497 & 0.0531 & 0.2008 & -0.1864 \\
1100 & -17.186 & -16.579 & -25.435 & -3.4593 & -0.2019 & -32.018 & 3.1768 & 0.0182 & 0.1931 & -0.2067 \\
1200 & -21.292 & -18.932 & -21.379 & -3.0945 & -0.1506 & -42.966 & 0.0363 & -0.0051 & 0.1917 & -0.1904 \\
1300 & -26.782 & -21.257 & -18.053 & -2.7730 & -0.0910 & -55.972 & -4.0071 & -0.0072 & 0.2058 & -0.1377 \\
1400 & -34.193 & -23.366 & -15.321 & -2.4898 & -0.0212 & -71.313 & -9.0779 & 0.0268 & 0.2493 & -0.0681 \\
1500 & -44.183 & -25.025 & -13.066 & -2.2405 & 0.0598 & -89.177 & -15.266 & 0.1140 & 0.3370 & 0.0141 \\
1600 & -57.540 & -25.924 & -11.196 & -2.0209 & 0.1528 & -109.72 & -22.625 & 0.2718 & 0.4888 & 0.1558 \\
1700 & -75.203 & -25.715 & -9.6367 & -1.8273 & 0.2584 & -133.11 & -31.192 & 0.5271 & 0.7308 & 0.3566 \\
1800 & -98.277 & -24.042 & -8.3357 & -1.6560 & 0.3769 & -159.48 & -40.991 & 0.9038 & 1.0907 & 0.5645 \\
1900 & -128.11 & -20.522 & -7.2402 & -1.5045 & 0.5089 & -189.04 & -52.100 & 1.4318 & 1.6084 & 0.8363 \\
2000 & -166.30 & -14.797 & -6.3199 & -1.3699 & 0.6537 & -222.20 & -64.787 & 2.1540 & 2.3542 & 1.1126 \\
\hline \hline
\end{tabular}

TABLE VII. Coefficients $\tilde{C}_{i}(q)$ of the $R_{L L}^{A A}$ response function in the semiempirical MEC formulas.

\begin{tabular}{|c|c|c|c|c|c|c|c|c|c|c|}
\hline q & $\tilde{C}_{1, A 1}$ & $\tilde{C}_{1, A 2}$ & $\tilde{C}_{2, A 1}$ & $\tilde{C}_{2, A 2}$ & $\tilde{C}_{3, A}$ & $\tilde{C}_{4, A 1}$ & $\tilde{C}_{4, A 2}$ & $\tilde{C}_{5, A 1}$ & $\tilde{C}_{5, A 2}$ & $\tilde{C}_{6, A}$ \\
\hline 200 & 7.7088 & 17.531 & 233.20 & 5.0374 & 9.4317 & 3.1687 & -49.648 & -1.2839 & 1.2848 & 3.4218 \\
\hline 300 & 10.129 & 9.2250 & 180.29 & 6.0908 & 8.1410 & -5.1516 & -17.177 & -1.1784 & 0.1189 & 2.1615 \\
\hline 400 & 10.742 & 11.672 & 130.90 & 6.3740 & 6.7068 & -5.1196 & -11.029 & -0.9983 & -0.4267 & 1.5098 \\
\hline 500 & 12.296 & 13.556 & 95.868 & 6.1594 & 5.5049 & -5.8216 & -8.7191 & -0.9352 & -0.6065 & 1.2160 \\
\hline 600 & 13.590 & 15.271 & 71.787 & 5.7313 & 4.5619 & -2.0130 & -8.5029 & -0.8949 & -0.6911 & 1.1226 \\
\hline 700 & 14.933 & 16.968 & 55.051 & 5.2304 & 3.8319 & 3.0303 & -8.0879 & -0.8569 & -0.7349 & 1.1446 \\
\hline 800 & 16.477 & 18.749 & 43.149 & 4.7251 & 3.2680 & 8.6441 & -7.2951 & -0.8243 & -0.7630 & 1.2170 \\
\hline 900 & 18.343 & 20.667 & 34.439 & 4.2463 & 2.8272 & 15.023 & -6.0701 & -0.8030 & -0.7775 & 1.3022 \\
\hline 1000 & 20.673 & 22.776 & 27.928 & 3.8069 & 2.4761 & 22.503 & -4.3435 & -0.8019 & -0.7903 & 1.3936 \\
\hline 1100 & 23.665 & 25.080 & 22.940 & 3.4113 & 2.1877 & 31.380 & -2.0133 & -0.8286 & -0.8101 & 1.4126 \\
\hline 1200 & 27.589 & 27.524 & 19.060 & 3.0580 & 1.9416 & 42.013 & 1.0546 & -0.8948 & -0.8527 & 1.4160 \\
\hline 1300 & 32.827 & 29.998 & 15.983 & 2.7448 & 1.7231 & 54.657 & 4.9885 & -1.0084 & -0.9300 & 1.3692 \\
\hline 1400 & 39.872 & 32.313 & 13.511 & 2.4679 & 1.5207 & 69.579 & 9.9124 & -1.1826 & -1.0605 & 1.2548 \\
\hline 1500 & 49.320 & 34.251 & 11.500 & 2.2234 & 1.3272 & 86.942 & 15.909 & -1.4322 & -1.2638 & 1.0643 \\
\hline 1600 & 61.902 & 35.509 & 9.8547 & 2.0074 & 1.1366 & 106.95 & 23.046 & -1.7716 & -1.5598 & 0.8203 \\
\hline 1700 & 78.500 & 35.763 & 8.4949 & 1.8163 & 0.9445 & 129.79 & 31.376 & -2.2227 & -1.9720 & 0.5642 \\
\hline 1800 & 100.17 & 34.688 & 7.3611 & 1.6472 & 0.7488 & 155.55 & 40.907 & -2.8139 & -2.5330 & 0.2057 \\
\hline 1900 & 128.23 & 31.939 & 6.4108 & 1.4973 & 0.5476 & 184.45 & 51.726 & -3.5732 & -3.2833 & -0.2214 \\
\hline 2000 & 164.22 & 27.217 & 5.6116 & 1.3640 & 0.3392 & 216.84 & 64.070 & -4.5204 & -4.2713 & -0.6653 \\
\hline
\end{tabular}


TABLE VIII. Coefficients $\tilde{C}_{i}(q)$ of the $R_{T^{\prime}}^{V A}$ response function in the semiempirical MEC formulas.

\begin{tabular}{lccccrrrrrr}
\hline \hline $\mathrm{q}$ & $\tilde{C}_{1, V A 1}$ & $\tilde{C}_{1, V A 2}$ & $\tilde{C}_{2, V A}$ & $\tilde{C}_{3, V A}$ & $\tilde{C}_{4, V A 1}$ & $\tilde{C}_{4, V A 2}$ & $\tilde{C}_{5, V A 1}$ & $\tilde{C}_{5, V A 2}$ & $\tilde{C}_{6, V A}$ & $\tilde{C}_{6, A V}$ \\
\hline 200 & 3.0775 & 7.0669 & 33.839 & 4.1248 & -122.58 & 175.36 & -0.28107 & 0.16341 & -84.995 & 135.39 \\
300 & 6.0856 & 7.0257 & 39.591 & 5.2873 & -45.017 & 68.720 & -0.36476 & -0.00935 & -5.9848 & 24.604 \\
400 & 8.2977 & 9.3332 & 40.713 & 5.6595 & 10.642 & -13.723 & -0.41794 & -0.17344 & 6.0440 & 6.4537 \\
500 & 10.413 & 12.083 & 39.821 & 5.6071 & 20.192 & -31.555 & -0.44973 & -0.30008 & 5.8446 & 5.3355 \\
600 & 12.682 & 15.166 & 38.151 & 5.3665 & 17.331 & -30.514 & -0.49020 & -0.39654 & 4.1826 & 6.3500 \\
700 & 14.967 & 18.484 & 36.245 & 5.0677 & 12.947 & -26.221 & -0.52323 & -0.46538 & 2.9376 & 6.8790 \\
800 & 17.214 & 21.943 & 34.390 & 4.7752 & 9.2326 & -22.152 & -0.53893 & -0.50361 & 2.1534 & 6.8773 \\
900 & 19.389 & 25.464 & 32.660 & 4.5129 & 6.4570 & -19.001 & -0.53699 & -0.51340 & 1.6290 & 6.6367 \\
1000 & 21.465 & 29.016 & 31.110 & 4.2871 & 4.4528 & -16.072 & -0.51836 & -0.49662 & 1.2863 & 6.2528 \\
1100 & 23.421 & 32.597 & 29.762 & 4.0941 & 3.0130 & -15.132 & -0.48558 & -0.45727 & 1.0445 & 5.8248 \\
1200 & 25.233 & 36.210 & 28.602 & 3.9272 & 1.9657 & -14.031 & -0.44114 & -0.39951 & 0.86212 & 5.3968 \\
1300 & 26.891 & 39.863 & 27.639 & 3.7794 & 1.1886 & -13.267 & -0.38738 & -0.32521 & 0.71212 & 4.9943 \\
1400 & 28.388 & 43.548 & 26.853 & 3.6443 & 0.59663 & -12.723 & -0.32579 & -0.23678 & 0.59011 & 4.6091 \\
1500 & 29.714 & 47.274 & 26.240 & 3.5167 & 0.13074 & -12.320 & -0.25785 & -0.13292 & 0.48717 & 4.2444 \\
1600 & 30.863 & 51.023 & 25.787 & 3.3926 & -0.24908 & -12.001 & -0.18497 & -0.01558 & 0.39962 & 3.8983 \\
1700 & 31.828 & 54.804 & 25.479 & 3.2691 & -0.57026 & -11.726 & -0.10735 & 0.11481 & 0.32699 & 3.5639 \\
1800 & 32.598 & 58.687 & 25.307 & 3.1443 & -0.85053 & -11.474 & -0.02521 & 0.25836 & 0.26413 & 3.2461 \\
1900 & 33.166 & 62.755 & 25.264 & 3.0170 & -1.1045 & -11.225 & 0.06088 & 0.41597 & 0.21063 & 2.9409 \\
2000 & 33.523 & 67.137 & 25.330 & 2.8868 & -1.3401 & -10.971 & 0.15142 & 0.59231 & 0.16352 & 2.6510 \\
\hline \hline
\end{tabular}

\section{APPENDIX C: PARAMETRIZATIONS OF NUCLEAR RESPONSE COEFFICIENTS}

In this Appendix, we provide parametrizations of the $q$ dependence of the semiempirical formula coefficients, $\tilde{C}_{i}(q)$, and of the averaged width, $\Gamma(q)$, and averaged shift, $\Sigma(q)$, of the averaged $\Delta$ propagator, $G_{\mathrm{av}}(q, \omega)$, for each one of the response functions in the semiempirical MEC formulas. We provide two kind of parametrizations. Most of the coefficients allow a polynomial parametrization,

$$
\tilde{C}_{i}(q)=\sum_{j=0}^{8} a_{j}\left(\frac{q}{2 m_{N}}\right)^{j}
$$

where the maximum range of the polynomial is 8 for some of the coefficients, but in many cases, the polynomial degree is less or much less than 8. The factors in the polynomial are dimensionless.

For some of the coefficients, the parametrization is better written as a polynomial of the inverse variable $2 m_{N} / q$,

$$
\tilde{C}_{i}(q)=\sum_{j=0}^{8} a_{j}\left(\frac{2 m_{N}}{q}\right)^{j}
$$

These are labeled with a star symbol (*) in the tables below.

The parameters of the averaged width, $\Gamma(q)$, and averaged shift, $\Sigma(q)$, are given in Table IX.

Parameters are provided in Tables $\mathrm{X}$ (for the $R_{T}^{V V}$ response function), XI (for $R_{T}^{A A}$ ), XII (for $R_{C C}^{V V}$ ), XIII (for $R_{C C}^{A A}$ ), XIV (for $R_{C L}^{A A}$ ), XV (for $R_{L L}^{A A}$ ), and XVI (for $R_{T^{\prime}}^{V A}$ ).

TABLE IX. Parameters of the effective shift, $\Sigma$, and width, $\Gamma$, of the averaged $\Delta$ propagator in the vector, axial, and vector-axial responses in the polynomial interpolations given by Eq. (C1), or Eq. (C2) for the ones labeled with a star symbol $*$.

\begin{tabular}{lccccccc}
\hline \hline & $a_{0}$ & \multicolumn{1}{c}{$a_{1}$} & \multicolumn{1}{c}{$a_{2}$} & $a_{3}$ & $a_{4}$ & $a_{5}$ & $a_{6}$ \\
\hline$\Sigma_{V}$ & 60.5191 & 174.869 & -142.624 & 0.0 & 64.8675 & 51.5966 & 0.0 \\
$\Gamma_{V}$ & 1450.93 & -14248.6 & 60480.2 & -132124 & 155882 & -94328.1 & 22938.6 \\
$\Sigma_{A} *$ & 93.7987 & -207.022 & 880.16 & -1497.82 & 1345.81 & -472.81 & 0.0 \\
$\Gamma_{A}$ & 48.5674 & 457.116 & -983.684 & 0.0 & 2539.27 & -3068.47 & 1104.8 \\
$\Sigma_{V A} *$ & 83.6648 & -43.5277 & 349.139 & -500.553 & 371.11 & -129.884 & 0.0 \\
$\Gamma_{V A} *$ & 173.605 & -566.125 & 2174.47 & -4916.66 & 5945.42 & -3691.91 & 935.097 \\
\hline \hline
\end{tabular}


TABLE X. Parameters of the coefficients $\tilde{C}_{i}(q)$ of the response function $R_{T}^{V V}$ in the interpolations given by Eq. (C1), or Eq. (C2) for the ones labeled with a star symbol $*$.

\begin{tabular}{lccccccccc}
\hline \hline & $a_{0}$ & $a_{1}$ & $a_{2}$ & $a_{3}$ & $a_{4}$ & $a_{5}$ & $a_{6}$ & $a_{7}$ \\
\hline$\tilde{C}_{1, V 1}$ & -19.6664 & 174.729 & -504.898 & 878.162 & -695.892 & 230.403 & 0.0 & 0.0 \\
$\tilde{C}_{1, V 2}$ & 0.0 & 253.264 & -2441.02 & 9243.9 & -14669.3 & 5825.93 & 10974.9 & -13547.1 & 4446.27 \\
$\tilde{C}_{2, V}$ & 464.634 & -1606.42 & 3069.48 & -2681.38 & 991.981 & 0.0 & 0.0 & 0.0 & 0.0 \\
$\tilde{C}_{3, V}$ & 0.0 & 10.3393 & 0.0 & 2.04851 & 0.0 & 0.0 & 0.0 & 0.0 & 0.0 \\
$\tilde{C}_{4, V 1}$ & 94.6881 & -1119.14 & 5033.78 & -11612.8 & 14309.6 & -8943.98 & 2231.48 & 0.0 & 0.0 \\
$\tilde{C}_{4, V 2}$ & -32.638 & 281.151 & -1007.61 & 1735.12 & -1334.5 & 390.521 & 0.0 & 0.0 & 0.0 \\
$\tilde{C}_{5, V 1}$ & 0.0 & -2.28139 & 4.09607 & -5.10766 & 0.0 & 0.0 & 0.0 & 0.0 & 0.0 \\
$\tilde{C}_{5, V 2}$ & 0.0 & 0.0 & -4.75457 & 0.0 & 12.9405 & -9.69383 & 0.0 & 0.0 & 0.0 \\
$\tilde{C}_{6, V}$ & -8.6321 & 188.024 & -702.668 & 1404.74 & -1543.33 & 882.04 & -205.234 & 0.0 & 0.0 \\
\hline \hline
\end{tabular}

TABLE XI. Parameters of the coefficients $\tilde{C}_{i}(q)$ of the response function $R_{T}^{A A}$ in the polynomial interpolations given by Eq. (C1), or Eq. (C2) for the ones labeled with a star symbol $*$.

\begin{tabular}{lccccccc}
\hline \hline & $a_{0}$ & $a_{1}$ & $a_{2}$ & $a_{3}$ & $a_{4}$ & $a_{5}$ & $a_{6}$ \\
\hline$\tilde{C}_{1, A 1} *$ & 40.1435 & -6.64283 & 0.0 & 0.354216 & -0.0596866 & 0.00286741 \\
$\tilde{C}_{1, A 2} *$ & 70.1364 & -18.7631 & 0.0 & 1.64276 & -0.448886 & 0.0478086 & -0.0017799 \\
$\tilde{C}_{2, A} *$ & -4.81568 & 8.57371 & -1.05139 & 0.0446577 & 0.0 & 0.0 & 0.0 \\
$\tilde{C}_{3, A} *$ & -1.70277 & 4.96453 & -0.275884 & 0.0 & 0.0 & 0.0 & 0.0 \\
$\tilde{C}_{4, A 1} *$ & -7.1971 & 8.61063 & -2.84088 & 0.399384 & -0.0192562 & 0.0 & 0.0 \\
$\tilde{C}_{4, A 2} *$ & 2.41265 & 1.1986 & -0.572642 & 0.0682446 & -0.00286093 & 0.0 \\
$\tilde{C}_{5, A 1} *$ & 3.60383 & -7.63457 & 3.56018 & -0.757029 & 0.0746929 & -0.00277361 \\
$\tilde{C}_{5, A 2} *$ & 0.564127 & -2.1691 & 0.636593 & -0.0652588 & 0.00261604 & 0.0 & 0.0 \\
$\tilde{C}_{6, A} *$ & -6.78958 & 6.19498 & -0.386024 & 0.0 & 0.0 & 0.0 \\
\hline \hline
\end{tabular}

TABLE XII. Parameters of the coefficients $\tilde{C}_{i}(q)$ of the response function $R_{C C}^{V V}$ in the polynomial interpolations given by Eq. (C1), or Eq. (C2) for the ones labeled with a star symbol $*$.

\begin{tabular}{|c|c|c|c|c|c|c|c|c|c|}
\hline & $a_{0}$ & $a_{1}$ & $a_{2}$ & $a_{3}$ & $a_{4}$ & $a_{5}$ & $a_{6}$ & $a_{7}$ & $a_{8}$ \\
\hline$\tilde{C}_{1, V 1}$ & 5.86212 & -159.987 & 1683.15 & -9120.25 & 28225.1 & -51256.7 & 53538.9 & -29301.5 & 6584.11 \\
\hline$\tilde{C}_{1, V 2}$ & 0.0 & 10.2676 & -141.292 & 839.978 & -2670.46 & 4936.0 & -5039.63 & 2519.75 & -504.43 \\
\hline$\tilde{C}_{2, V}$ & 13.7234 & -61.873 & 132.704 & -119.937 & 39.5931 & 0.0 & 0.0 & 0.0 & 0.0 \\
\hline$\tilde{C}_{3, V}$ & -0.0686547 & 0.0 & 8.18253 & -2.41841 & 0.0 & 0.0 & 0.0 & 0.0 & 0.0 \\
\hline$\tilde{C}_{4, V 1}$ & 1.75335 & -23.1728 & 127.716 & -386.742 & 628.412 & -421.798 & 107.647 & 0.0 & 0.0 \\
\hline$\tilde{C}_{4, V 2}$ & 0.0 & -4.25877 & 36.7999 & -123.11 & 166.971 & -60.689 & 0.0 & 0.0 & 0.0 \\
\hline$\tilde{C}_{5, V 1}$ & 0.0 & -1.50012 & 8.60699 & -14.8142 & 0.0 & 0.0 & 0.0 & 0.0 & 0.0 \\
\hline$\tilde{C}_{5, V 2}$ & 0.0 & 0.542587 & -6.74484 & 22.0466 & -29.4463 & 6.74147 & 0.0 & 0.0 & 0.0 \\
\hline$\tilde{C}_{6, V}$ & 0.0 & -0.288279 & 5.91647 & 0.0 & -10.6428 & 9.91934 & -2.86346 & 0.0 & 0.0 \\
\hline
\end{tabular}


TABLE XIII. Parameters of the coefficients $\tilde{C}_{i}(q)$ of the response function $R_{C C}^{A A}$ in the polynomial interpolations given by Eq. (C1), or Eq. (C2) for the ones labeled with a star symbol $*$.

\begin{tabular}{|c|c|c|c|c|c|c|c|}
\hline & $a_{0}$ & $a_{1}$ & $a_{2}$ & $a_{3}$ & $a_{4}$ & $a_{5}$ & $a_{6}$ \\
\hline$\tilde{C}_{1, A 1}$ & 1.00967 & -13.0344 & 73.0221 & 0.0 & -198.704 & 259.352 & 0.0 \\
\hline$\tilde{C}_{1, A 2}$ & 3.83926 & -62.2338 & 400.672 & -1071.58 & 1612.63 & -1160.11 & 288.513 \\
\hline$\tilde{C}_{2, A 1} *$ & -39.489 & 44.8644 & 1.70487 & -0.355919 & 0.0 & 0.0 & 0.0 \\
\hline$\tilde{C}_{2, A 2} *$ & -2.17605 & 4.26239 & -0.589857 & 0.0284662 & 0.0 & 0.0 & 0.0 \\
\hline$\tilde{C}_{3, A}$ & 0.491785 & -1.6485 & 4.50652 & -5.29804 & 1.47136 & 0.0 & 0.0 \\
\hline$\tilde{C}_{4, A 1}$ & 0.0 & -39.1279 & 69.8619 & 155.687 & 0.0 & 0.0 & 0.0 \\
\hline$\tilde{C}_{4, A 2}$ & -237.745 & 3006.35 & -14949.7 & 36613.0 & -47206.4 & 30846.5 & -8019.86 \\
\hline$\tilde{C}_{5, A 1}$ & 0.0888813 & -0.283259 & -1.87097 & 7.84059 & 0.0 & -5.55097 & 0.0 \\
\hline$\tilde{C}_{5, A 2}$ & -0.408277 & 5.44882 & -22.9732 & 37.91 & -20.0388 & 0.0 & 0.0 \\
\hline$\tilde{C}_{6, A}$ & 1.04713 & -8.02409 & 14.0568 & -12.9651 & 3.55436 & 0.0 & 0.0 \\
\hline
\end{tabular}

TABLE XIV. Parameters of the coefficients $\tilde{C}_{i}(q)$ of the response function $R_{C L}^{A A}$ in the polynomial interpolations given by Eq. (C1), or Eq. (C2) for the ones labeled with a star symbol $*$.

\begin{tabular}{|c|c|c|c|c|c|c|c|}
\hline & $a_{0}$ & $a_{1}$ & $a_{2}$ & $a_{3}$ & $a_{4}$ & $a_{5}$ & $a_{6}$ \\
\hline$\tilde{C}_{1, A 1}$ & 0.0 & -5.53339 & -53.5517 & 0.0 & 177.554 & -239.114 & 0.0 \\
\hline$\tilde{C}_{1, A 2}$ & -9.61627 & 139.949 & -899.062 & 2402.54 & -3417.33 & 2375.71 & -613.648 \\
\hline$\tilde{C}_{2, A 1} *$ & 0.0 & 11.7941 & -25.2288 & 6.74752 & -0.71334 & 0.0274023 & 0.0 \\
\hline$\tilde{C}_{2, A 2} *$ & 2.15609 & -4.27799 & 0.628945 & -0.0298366 & 0.0 & 0.0 & 0.0 \\
\hline$\tilde{C}_{3, A}$ & 0.636771 & -7.06185 & 17.1626 & -16.5615 & 6.28956 & 0.0 & 0.0 \\
\hline$\tilde{C}_{4, A 1}$ & 0.0 & 56.6646 & -111.747 & -126.814 & 0.0 & 0.0 & 0.0 \\
\hline$\tilde{C}_{4, A 2}$ & 136.9 & -1630.92 & 7986.45 & -19326.8 & 24651.6 & -16013.6 & 4146.06 \\
\hline$\tilde{C}_{5, A 1}$ & 0.0 & 1.19682 & -1.25693 & -2.63388 & 0.0 & 3.99421 & 0.0 \\
\hline$\tilde{C}_{5, A 2}$ & 0.325959 & -3.9354 & 18.934 & -32.2136 & 18.3759 & 0.0 & 0.0 \\
\hline$\tilde{C}_{6, A}$ & -1.6945 & 10.4321 & -24.2097 & 21.6826 & -5.45423 & 0.0 & 0.0 \\
\hline
\end{tabular}

TABLE XV. Parameters of the coefficients $\tilde{C}_{i}(q)$ of the response function $R_{L L}^{A A}$ in the polynomial interpolations given by Eq. $(\mathrm{C} 1)$, or Eq. (C2) for the ones labeled with a star symbol $*$.

\begin{tabular}{|c|c|c|c|c|c|c|c|c|c|}
\hline & $a_{0}$ & $a_{1}$ & $a_{2}$ & $a_{3}$ & $a_{4}$ & $a_{5}$ & $a_{6}$ & $a_{7}$ & $a_{8}$ \\
\hline$\tilde{C}_{1, A 1}$ & 6.14051 & 16.8939 & 26.5549 & 0.0 & -131.934 & 203.693 & 0.0 & 0.0 & 0.0 \\
\hline$\tilde{C}_{1, A 2}$ & 141.573 & -2569.7 & 19929.8 & -81032.4 & 193118 & -278723 & 239909 & -113349 & 22609.3 \\
\hline$\tilde{C}_{2, A 1} *$ & -12.4591 & 12.5106 & 6.0512 & -0.490028 & 0.0 & 0.0 & 0.0 & 0.0 & 0.0 \\
\hline$\tilde{C}_{2, A 2} *$ & -2.2448 & 4.431 & -0.710191 & 0.0341804 & 0.0 & 0.0 & 0.0 & 0.0 & 0.0 \\
\hline$\tilde{C}_{3, A}$ & 13.7017 & -45.4469 & 66.7382 & -45.3372 & 10.9484 & 0.0 & 0.0 & 0.0 & 0.0 \\
\hline$\tilde{C}_{4, A 1}$ & 0.0 & -34.6322 & 55.3018 & 156.919 & 0.0 & 0.0 & 0.0 & 0.0 & 0.0 \\
\hline$\tilde{C}_{4, A 2}$ & -204.783 & 2442.11 & -11760.5 & 28204.8 & -35811.5 & 23149.8 & -5969.69 & 0.0 & 0.0 \\
\hline$\tilde{C}_{5, A 1}$ & -1.63891 & 4.02606 & -6.87094 & 5.50556 & 0.0 & -4.39721 & 0.0 & 0.0 & 0.0 \\
\hline$\tilde{C}_{5, A 2}$ & 3.52402 & -29.492 & 67.8688 & -60.0326 & 14.9714 & 0.0 & 0.0 & 0.0 & 0.0 \\
\hline$\tilde{C}_{6, A}$ & 6.74744 & -43.2203 & 113.243 & -117.526 & 40.5894 & 0.0 & 0.0 & 0.0 & 0.0 \\
\hline
\end{tabular}


TABLE XVI. Parameters of the coefficients $\tilde{C}_{i}(q)$ of the response function $R_{T^{\prime}}^{V A}$ in the polynomial interpolations given by Eq. (C1), or Eq. (C2) for the ones labeled with a star symbol $*$.

\begin{tabular}{|c|c|c|c|c|c|c|c|c|c|}
\hline & $a_{0}$ & $a_{1}$ & $a_{2}$ & $a_{3}$ & $a_{4}$ & $a_{5}$ & $a_{6}$ & $a_{7}$ & $a_{8}$ \\
\hline$\tilde{C}_{1, V A 1}$ & -12.5654 & 271.856 & -1772.34 & 7170.88 & -16850.5 & 23866.6 & -20160.5 & 9354.45 & -1834.88 \\
\hline$\tilde{C}_{1, V A 2}$ & 25.7693 & -401.628 & 3111.18 & -11803.4 & 27278.8 & -38962.8 & 33541.9 & -15937.6 & 3209.45 \\
\hline$\tilde{C}_{2, V A}$ & 0.981663 & 516.73 & -2461.18 & 5506.71 & -6578.11 & 4042.59 & -1002.31 & 0.0 & 0.0 \\
\hline$\tilde{C}_{3, V A}$ & -0.698901 & 70.138 & -272.492 & 456.781 & -355.974 & 105.235 & 0.0 & 0.0 & 0.0 \\
\hline$\tilde{C}_{4, V A 1} *$ & -12.6139 & 27.5451 & -27.9095 & 15.1272 & -3.53758 & 0.346934 & -0.0120447 & 0.0 & 0.0 \\
\hline$\tilde{C}_{4, V A 2} *$ & -21.627 & 21.438 & -11.7492 & 0.0 & 0.549239 & -0.0443672 & 0.0 & 0.0 & 0.0 \\
\hline$\tilde{C}_{5, V A 1}$ & -0.239117 & 0.0 & -6.79621 & 17.8376 & -15.5393 & 4.77589 & 0.0 & 0.0 & 0.0 \\
\hline$\tilde{C}_{5, V A 2}$ & 0.570313 & -3.93619 & 0.0 & 12.945 & -14.1847 & 4.9816 & 0.0 & 0.0 & 0.0 \\
\hline$\tilde{C}_{6, V A} *$ & -3.54219 & 8.09376 & -6.67611 & 2.807 & -0.500736 & 0.035343 & -0.000846361 & 0.0 & 0.0 \\
\hline$\tilde{C}_{6, A V^{*}}$ & -0.47772 & 0.0 & 6.24771 & -3.31642 & 0.616557 & -0.0426815 & 0.000953283 & 0.0 & 0.0 \\
\hline
\end{tabular}

[1] H. Gallagher, G. Garvey, and G. P. Zeller, Annu. Rev. Nucl. Part. Sci. 61, 355 (2011).

[2] J. G. Morfin, J. Nieves, and J. T. Sobczyk, Adv. High Energy Phys. 2012, 35 (2012).

[3] J. A. Formaggio and G. P. Zeller, Rev. Mod. Phys. 84, 1307 (2012).

[4] L. Alvarez-Ruso, Y. Hayato, and J. Nieves, New J. Phys. 16, 075015 (2014).

[5] U. Mosel, Annu. Rev. Nucl. Part. Sci. 66, 171 (2016).

[6] A. M. Ankowski and C. Mariani, J. Phys. G 44, 054001 (2017).

[7] O. Benhar, P. Huber, C. Mariani, and D. Meloni, Phys. Rep. 700, 1 (2017).

[8] T. Katori and M. Martini, J. Phys. G 45, 013001 (2018).

[9] J. E. Amaro, M. B. Barbaro, J. A. Caballero, R. GonzálezJiménez, G. D. Megias, and I. Ruiz Simo, J. Phys. G 47, 124001 (2020).

[10] V Lyubushkin et al. (NOMAD Collaboration), Eur. Phys. J. C 63, 355 (2009).

[11] A. Aguilar-Arevalo et al. (MiniBooNE Collaboration), Phys. Rev. D 81, 092005 (2010).

[12] A. Aguilar-Arevalo et al. (MiniBooNE Collaboration), Phys. Rev. D 88, 032001 (2013).

[13] G. A. Fiorentini et al. (MINERvA Collaboration), Phys. Rev. Lett. 111, 022502 (2013).

[14] K. Abe et al. (T2K Collaboration), Phys. Rev. D 87, 092003 (2013).

[15] K. Abe et al. (T2K Collaboration), Phys. Rev. D 93, 112012 (2016).

[16] K. Abe et al. (T2K Collaboration), Phys. Rev. D 97, 012001 (2018).

[17] M. Martini, M. Ericson, G. Chanfray, and J. Marteau, Phys. Rev. C 80, 065501 (2009).

[18] J. Nieves, I. Ruiz Simo, and M. J. Vicente Vacas, Phys. Rev. C 83, 045501 (2011).

[19] K. Gallmeister, U. Mosel, and J. Weil, Phys. Rev. C 94, 035502 (2016).
[20] G. D Megias, J. E. Amaro, M. B. Barbaro, J. A. Caballero, T. W. Donnelly, and I. Ruiz Simo, Phys. Rev. D 94, 093004 (2016).

[21] G. D. Megias, M. V. Ivanov, R. Gonzalez-Jimenez, M. B. Barbaro, J. A. Caballero, T. W. Donnelly, and J. M. Udias, Phys. Rev. D 89, 093002 (2014); 91, 039903(E) (2015).

[22] A. M. Ankowski, Phys. Rev. D 92, 013007 (2015).

[23] R. Gran, J. Nieves, F. Sanchez, and M. J. Vicente Vacas, Phys. Rev. D 88, 113007 (2013).

[24] V. Pandey, N. Jachowicz, M. Martini, R. Gonzalez-Jimenez, J. Ryckebusch, T. Van Cuyck, and N. Van Dessel, Phys. Rev. C 94, 054609 (2016).

[25] M. Martini, N. Jachowicz, M. Ericson, V. Pandey, T. Van Cuyck, and N. Van Dessel, Phys. Rev. C 94, 015501 (2016).

[26] A. Lovato, S. Gandolfi, J. Carlson, Steven C. Pieper, and R. Schiavilla, Phys. Rev. Lett. 117, 082501 (2016).

[27] V. Pandey, N. Jachowicz, T. Van Cuyck, J. Ryckebusch, and M. Martini, Phys. Rev. C 92, 024606 (2015).

[28] A. M. Ankowski, O. Benhar, and M. Sakuda, Phys. Rev. D 91, 033005 (2015).

[29] N. Rocco, A. Lovato, and O. Benhar, Phys. Rev. Lett. 116, 192501 (2016).

[30] J. E. Amaro, M. B. Barbaro, J. A. Caballero, T. W. Donnelly, and A. Molinari, Phys. Rep. 368, 317 (2002).

[31] J. E. Amaro, M. B. Barbaro, J. A. Caballero, T. W. Donnelly, and C. Maieron, Phys. Rev. C 71, 065501 (2005).

[32] J. A. Caballero, J. E. Amaro, M. B. Barbaro, T. W. Donnelly, and J. M. Udias, Phys. Lett. B 653, 366 (2007).

[33] J. M. Udias, J. A. Caballero, E. Moya de Guerra, J. E. Amaro, and T. W. Donnelly, Phys. Rev. Lett. 83, 5451 (1999).

[34] A. Bodek, M. E. Christy, and B. Coopersmith, Eur. Phys. J. C 74, 3091 (2014).

[35] J. E. Sobczyk, Phys. Rev. C 96, 045501 (2017).

[36] M. Martini, M. Ericson, G. Chanfray, and J. Marteau, Phys. Rev. C 81, 045502 (2010). 
[37] J. E. Amaro, M. B. Barbaro, J. A. Caballero, T. W. Donnelly, and C. F. Williamson, Phys. Lett. B 696, 151 (2011).

[38] J. E. Amaro, M. B. Barbaro, J. A. Caballero, and T. W. Donnelly, Phys. Rev. Lett. 108, 152501 (2012).

[39] J. Nieves, I. Ruiz Simo, and M. J. Vicente Vacas, Phys. Lett. B 707, 72 (2012).

[40] J. Nieves, I. Ruiz Simo, and M. J. Vicente Vacas, Phys. Lett. B 721, 90 (2013).

[41] I. Ruiz Simo, J. E. Amaro, M. B. Barbaro, J. A. Caballero, G. D. Megias, and T. W. Donnelly, Phys. Lett. B 770, 193 (2017).

[42] I. Ruiz Simo, J. E. Amaro, M. B. Barbaro, J. A. Caballero, G. D. Megias, and T. W. Donnelly, Ann. Phys. (Amsterdam) 388, 323 (2018).

[43] J. E. Sobczyk, J. Nieves, and F. Sánchez, Phys. Rev. C 102, 024601 (2020).

[44] S. Dolan, G. D. Megias, and S. Bolognesi, Phys. Rev. D 101, 033003 (2020).

[45] O. Lalakulich, K. Gallmeister, and U. Mosel, Phys. Rev. C 86, 014614 (2012); 90, 029902(E) (2014).

[46] G. D. Megias, T. W. Donnelly, O. Moreno, C. F. Williamson, J. A. Caballero, R. González-Jiménez, A. De Pace, M. B. Barbaro, W. M. Alberico, M. Nardi, and J. E. Amaro, Phys. Rev. D 91, 073004 (2015).

[47] C. F. von Weizsäcker, Z. Phys. A Hadrons Nucl. 96, 431 (1935).

[48] J. E. Amaro, E. Ruiz Arriola, and I. Ruiz Simo, Phys. Rev. C 92, 054607 (2015).

[49] J. E. Amaro, E. Ruiz Arriola, and I. Ruiz Simo, Phys. Rev. D 95, 076009 (2017).

[50] V. L. Martinez-Consentino, I. Ruiz Simo, J. E. Amaro, and E. Ruiz Arriola, Phys. Rev. C 96, 064612 (2017).

[51] I. Ruiz Simo, V. L. Martinez-Consentino, J. E. Amaro, and E. Ruiz Arriola, Phys. Rev. D 97, 116006 (2018).

[52] J. E. Amaro, V. L. Martinez-Consentino, E. Ruiz Arriola, and I. Ruiz Simo, Phys. Rev. C 98, 024627 (2018).

[53] V. L. Martinez-Consentino, I. R. Simo, and J. E. Amaro, Phys. Rev. C 104, 025501 (2021).

[54] R. Rosenfelder, Ann. Phys. (N.Y.) 128, 188 (1980).
[55] B. D. Serot and J. D. Walecka, in Advances in Nuclear Physics, edited by J.W. Negele and E. Vogt (Plenum, New York, 1986), Vol. 16.

[56] K. Wehrberger, Phys. Rep. 225, 273 (1993).

[57] M. B. Barbaro, R. Cenni, A. De Pace, T. W. Donnelly, and A. Molinari, Nucl. Phys. A643, 137 (1998).

[58] I. Ruiz Simo, C. Albertus, J. E. Amaro, M. B. Barbaro, J. A. Caballero, and T. W. Donnelly, Phys. Rev. D 90, 033012 (2014).

[59] E. Hernandez, J. Nieves, and M. Valverde, Phys. Rev. D 76, 033005 (2007).

[60] J. E. Amaro, C. Maieron, M. B. Barbaro, J. A. Caballero, and T. W. Donnelly, Phys. Rev. C 82, 044601 (2010).

[61] T. Van Cuyck, N. Jachowicz, R. González-Jiménez, M. Martini, V. Pandey, J. Ryckebusch, and N. Van Dessel, Phys. Rev. C 94, 024611 (2016).

[62] B. Sommer, Nucl. Phys. A308, 263 (1978).

[63] W. Alberico, M. Ericson, and A. Molinari, Ann. Phys. (N.Y.) 154, 356 (1984).

[64] S. Galster, H. Klein, J. Moritz, K. H. Schmidt, D. Wegener, and J. Bleckwenn, Nucl. Phys. B32, 221 (1971).

[65] M. J. Dekker, P. J. Brussaard, and J. A. Tjon, Phys. Rev. C 49, 2650 (1994).

[66] I. Ruiz Simo, J. E. Amaro, M. B. Barbaro, A. De Pace, J. A. Caballero, and T. W. Donnelly, J. Phys. G 44, 065105 (2017).

[67] I. Ruiz Simo, C. Albertus, J. E. Amaro, M. B. Barbaro, J. A. Caballero, and T. W. Donnelly, Phys. Rev. D 90, 053010 (2014).

[68] Proteus scientific computing cloud, https://proteus.ugr.es/ en/proteus-eng/.

[69] O. Benhar, D. Day, and I. Sick, arXiv:nucl-ex/0603032.

[70] O. Benhar, D. Day, and I. Sick, http://faculty.virginia.edu/ qes-archive/.

[71] O. Benhar, D. Day, and I. Sick, Rev. Mod. Phys. 80, 189 (2008).

[72] H. C. Kim, S. Schramm, and C. J. Horowitz, Phys. Rev. C 53, 2468 (1996).

[73] See Supplemental Material at http://link.aps.org/ supplemental/10.1103/PhysRevD.104.113006 for text files with the parameters of Appendices B and C. 